\title{
Primary frequency regulation with load-side participation Part I: stability and optimality
}

\author{
Andreas Kasis, Eoin Devane, Chrysovalantis Spanias, and Ioannis Lestas
}

\begin{abstract}
We present a method to design distributed generation and demand control schemes for primary frequency regulation in power networks that guarantee asymptotic stability and ensure fairness of allocation. We impose a passivity condition on net power supply variables and provide explicit steady state conditions on a general class of generation and demand control dynamics that ensure convergence of solutions to equilibria that solve an appropriately constructed network optimization problem. We also show that the inclusion of controllable demand results in a drop in steady state frequency deviations. We discuss how various classes of dynamics used in recent studies fit within our framework and show that this allows for less conservative stability and optimality conditions. We illustrate our results with simulations on the IEEE 68-bus transmission system and the IEEE 37-bus distribution system with static and dynamic demand response schemes.
\end{abstract}

Index Terms-frequency control, stability, optimization

\section{INTRODUCTION}

Large scale integration of renewable sources of energy within the power grid is expected to cause fast changes in generation, making power imbalances increasingly frequent due to the inability of conventional means of generation to counter-balance them [2], [3]. Load participation is considered to be one potential solution to this problem, providing fast response to power changes. Household appliances like air conditioning units, heaters, and refrigerators can be controlled to adjust frequency and regulate power imbalances. Although the idea dates back to the 1970s [4], research attention has recently increasingly focused on the concept of controllable demand [5], [6], [7] with particular consideration given to its use for primary control [8], [9]. Frequency control in a conventional power grid is implemented by means of control schemes that operate at different timescales [10], [11], [12]. Primary or droop control operates at a timescale of up to about 10 seconds and is a decentralized scheme providing real-time balancing of supply and demand. Secondary control operates at a timescale of minutes and recovers the frequency to its nominal value. Issues of optimality are addressed via tertiary

Paper [1] is a preliminary conference version of this manuscript. This manuscript includes proofs of our main results, additional results associated with stability and steady state behaviour, and also additional discussion.

The research carried out was partly funded by an ERC starting grant.

Andreas Kasis is with the Department of Engineering, University of Cambridge, Trumpington Street, Cambridge, CB2 1PZ, United Kingdom; email: ak647@cam.ac.uk

Eoin Devane is with the Cambridge Centre for Analysis, Centre for Mathematical Sciences, University of Cambridge, Wilberforce Road, Cambridge, CB3 0WA, United Kingdom; e-mail: esmd2@cam.ac.uk

Chrysovalantis Spanias is with the Department of Electrical Engineering, Computer Engineering and Informatics, Cyprus University of Technology, Limassol, Cyprus; email: ca.spanias@edu.cut.ac.cy

Ioannis Lestas is with the Department of Engineering, University of Cambridge, Trumpington Street, Cambridge, CB2 1PZ, United Kingdom;; e-mail: icl20@cam.ac.uk control schemes at a much slower timescale ranging from about 15 minutes to several hours.

An issue of fairness and optimality in the allocation is, however, raised if highly distributed schemes are to be used for frequency control at faster timescales, such as schemes involving controllable loads. Recent studies have attempted to address this issue by devising control schemes which solve an optimization problem guaranteeing a fair allocation between them. This approach has been studied for primary and also for secondary control. We consider here primary rather than secondary control in order to avoid the additional communication that would be necessary to get a fair allocation if controllable demand were used in the latter case. This is because it is evident that a synchronizing variable is necessary to achieve optimality, allowing all nodes to adapt their generation and controllable demand so as to attain equal marginal costs. In primary control, frequency deviation from the nominal value can be used for this purpose, allowing decentralized control to be achieved [13], [14], [15]. In secondary control, where frequency deviations return to zero, a different variable needs to be used for synchronization. This is often achieved by additional information exchange between neighboring buses [16], [17], [18], [19] or via special network structures such as star topologies [20]. It should be noted that communication of additional information allows also additional constraints to be satisfied, such as line constraints [19].

In this paper, we consider a network model described by nonlinear swing equations. We consider a general class of dynamics for power generation and controllable demand, on which we impose appropriate conditions so as to achieve stability of the equilibrium points and an optimization interpretation of those. This allows us to guarantee, for a wide variety of possible generation and demand dynamics, convergence to a power allocation that solves an appropriately constructed optimization problem, thus ensuring fairness in this allocation. The class of dynamics considered incorporates control schemes using only local frequency measurements as input signals, and we demonstrate that this is sufficient to enable them to take the right decisions so as to converge to a global optimum, thus allowing distributed control. We illustrate the applicability of our approach by demonstrating that various dynamics that have been used in recent interesting studies, such as [13] and [14], can be incorporated within our framework, and we show that the analysis presented in the paper can give less conservative stability and optimality conditions.

It should be noted that one of the distinctive features of our analysis is that optimality of the power allocation is provided via appropriate conditions on the input/output properties of the systems considered. In our companion paper [21] we show how additional local information can be exploited to relax a 
passivity condition used in this paper to deduce convergence.

The paper is organized as follows. Sections II and III give some basic notation and preliminaries. In Section IV, we present the power network model and section $\mathrm{V}$ presents our main results, which are proved in Appendix A. In Section VI, we discuss how our analysis relates to other important studies. Section VII illustrates our results through simulations on the IEEE 68-bus transmission system and the IEEE 37-bus distribution system. Finally, conclusions are drawn in Section VIII.

\section{NOTATION}

For a function $f(q)$, we denote its first derivative by $f^{\prime}(q)=$ $\frac{d}{d q} f(q)$. The expression $f^{-1}(w)$ represents the preimage of the point $w$ under the function $f$, i.e. $f^{-1}(w)=\{q: f(q)=w\}$. When the function $f$ is invertible, $f^{-1}$ then defines the inverse function of $f$. A function $f: \mathbb{R}^{n} \rightarrow \mathbb{R}$ is said to be positive definite on a neighbourhood $D$ around the origin if $f(0)=0$ and $f(x)>0$ for every non-zero $x \in D$. It is positive semidefinite if the inequality $>0$ is replaced by $\geq 0$. Furthermore, $[q]_{a}^{b}$ denotes $\max \{\min \{q, b\}, a\}$ for $a, b \in \mathbb{R}, a \leq b$. The indicator function $\mathbb{1}_{S}: \mathbb{R}^{n} \rightarrow\{0,1\}$ of a set $S \subseteq \mathbb{R}^{n}$ takes the value 1 if its argument belongs to the set $S$ and 0 otherwise. We denote the derivative of a function $q(t)$ with respect to time by $\dot{q}$ and its Laplace transform by $\hat{q}=\int_{0}^{\infty} e^{-s t} q(t) d t$. For a system as in (1) where $x=\bar{x}, u=y=0$ is an equilibrium point, the $\mathcal{L}_{2}$-gain is defined as $\sup _{\|u\|_{2} \neq 0} \frac{\|y\|_{2}}{\|u\|_{2}}$ with $x(0)=\bar{x}$, where the $\mathcal{L}_{2}$-norm is $\|f\|_{2}=\sqrt{\int_{0}^{\infty} f^{2}(t) d t}$. It can be shown that for a stable linear system with transfer function $G(s)$ its $\mathcal{L}_{2}$-gain is given by $\sup _{\phi}|G(j \phi)|[22]$.

\section{PRELIMINARIES}

Throughout the paper we will consider dynamical systems with input $u(t) \in \mathbb{R}$, state $x(t) \in \mathbb{R}^{m}$, and output $y(t) \in \mathbb{R}$ with a state space realization of the form

$$
\begin{aligned}
& \dot{x}=f(x, u), \\
& y=g(x, u),
\end{aligned}
$$

where $f: \mathbb{R}^{m} \times \mathbb{R} \rightarrow \mathbb{R}^{m}$ is locally Lipschitz and $g: \mathbb{R}^{m} \times$ $\mathbb{R} \rightarrow \mathbb{R}$ is continuous. We assume in system (1) that given any constant input $u(t) \equiv \bar{u} \in \mathbb{R}$, there exists a unique locally asymptotically stable equilibrium point $\bar{x} \in \mathbb{R}^{m}$, i.e. $f(\bar{x}, \bar{u})=$ 0 . The region of attraction ${ }^{1}$ of $\bar{x}$ is denoted by $X_{0}$. We also define the static input-state characteristic map $k_{x}: \mathbb{R} \rightarrow \mathbb{R}^{m}$,

$$
k_{x}(\bar{u}):=\bar{x} .
$$

Based on this, we can also define the static input-output characteristic map $k_{y}: \mathbb{R} \rightarrow \mathbb{R}$,

$$
k_{y}(\bar{u}):=g\left(k_{x}(\bar{u}), \bar{u}\right) .
$$

The requirement that for each constant input to (1) there exists a unique equilibrium point could be relaxed to require only isolated equilibria, however, we assume it here to simplify the presentation.

\footnotetext{
${ }^{1}$ That is, for the constant input $u=\bar{u}$, any solution $x(t)$ of (1) with initial condition $x(0) \in X_{0}$ must satisfy $x(t) \rightarrow \bar{x}$ as $t \rightarrow \infty$. The definition of local asymptotic stability also implies that $X_{0}$ has nonempty interior.
}

\begin{tabular}{ll}
$\omega_{j}$ & frequency \\
$\eta_{i j}$ & power angle difference between bus $i$ and bus $j$ \\
$p_{j}^{M}$ & mechanical power injection \\
$d_{j}^{c}$ & controllable load \\
$d_{j}^{u}$ & uncontrollable frequency dependent load \\
$p_{i j}$ & power transfer from bus $i$ to bus $j$ \\
$B_{i j}$ & line susceptance \\
$p_{j}^{L}$ & step change in uncontrollable demand \\
$x^{M, j}$ & internal states of generation dynamics \\
$x^{c, j}$ & internal states of controllable load dynamics \\
$x^{u, j}$ & internal states of uncontrollable frequency \\
& dependent load dynamics \\
\hline
\end{tabular}

Fig. 1. Notation used in the system model (3)-(4). Note that variables $\omega_{j}$, $p_{j}^{M}, d_{j}^{c}, d_{j}^{u}, p_{j}^{L}$ denote deviations from corresponding nominal values. Also by internal states we refer to the states in the state space representation of the differential equations representing the dynamics (details can be found in sections III and IV).

\section{PROBLEM FORMULATION}

\section{A. Network model}

The power network model is described by a graph $(N, E)$ where $N=\{1,2, \ldots,|N|\}$ is the set of buses and $E \subseteq N \times N$ is the set of transmission lines connecting the buses. There are two types of buses in the network, buses with inertia and buses without inertia. Let $G$ and $L$ be the sets of buses with and without inertia respectively such that $|G|+|L|=|N|$. Furthermore, we use $(i, j)$ to denote the link connecting buses $i$ and $j$ and assume that the graph $(N, E)$ is directed ${ }^{2}$ with arbitrary direction, so that if $(i, j) \in E$ then $(j, i) \notin E$. For each $j \in N$, we use $i: i \rightarrow j$ and $k: j \rightarrow k$ to denote the sets of buses that are predecessors and successors of bus $j$ respectively. It is important to note that the form of the dynamics in (3)-(4) below is unaltered by any change in the graph ordering, and all of our results are independent of the direction. We also assume that $(N, E)$ is connected and that: 1) Bus voltage magnitudes are $\left|V_{j}\right|=1$ p.u. for all $j \in N$.

2) Lines $(i, j) \in E$ are lossless and characterized by their susceptances $B_{i j}=B_{j i}>0$.

3) Reactive power flows do not affect bus voltage phase angles and frequencies.

These assumptions are frequently used in the literature to simplify the analysis, and usually hold at higher voltages and when the voltage within the system is tightly controlled.

The rate of change of frequency can then be represented using swing equations, while power must be conserved at each of the buses without inertia. This motivates the following system dynamics (e.g. [10])

$$
\begin{gathered}
\dot{\eta}_{i j}=\omega_{i}-\omega_{j},(i, j) \in E, \\
M_{j} \dot{\omega}_{j}=-p_{j}^{L}+p_{j}^{M}-\left(d_{j}^{c}+d_{j}^{u}\right)-\sum_{k: j \rightarrow k} p_{j k}+\sum_{i: i \rightarrow j} p_{i j}, j \in G,
\end{gathered}
$$

\footnotetext{
${ }^{2}$ It should be noted that the power transfer between buses is bidirectional, i.e. if $p_{i j}$ is the power transfer from $i$ to $j$ then the power transfer from $j$ to $i$ is $-p_{i j}$. The notion of a directed graph is only used here to facilitate notation so that a single variable, $p_{i j}$ or $p_{j i}$, is defined for each pair of buses.
} 


$$
\begin{gathered}
0=-p_{j}^{L}-\left(d_{j}^{c}+d_{j}^{u}\right)-\sum_{k: j \rightarrow k} p_{j k}+\sum_{i: i \rightarrow j} p_{i j}, j \in L, \\
p_{i j}=B_{i j} \sin \eta_{i j}-p_{i j}^{n o m},(i, j) \in E .
\end{gathered}
$$

In system (3) $p_{j}^{M}, \omega_{j}, d_{j}^{c}$ and $d_{j}^{u}$ are time-dependent variables representing, respectively, deviations from a nominal value ${ }^{3}$ for the following quantities: the mechanical power injection to the generator bus $j$, the frequency at any bus $j$, the controllable load and uncontrollable frequency-dependent load present at any bus $j$. The variables $\eta_{i j}$ and $p_{i j}$ represent, respectively, the power angle difference ${ }^{4}$ and the deviation from a nominal value $p_{i j}^{n o m}$ for the power transmitted from bus $i$ to bus $j$. The constant $M_{j}>0$ denotes the inertia at bus $j$. The variable $p_{j}^{L}$ denotes the deviation from a nominal value of a step change in the uncontrollable demand or generation at bus $j$. It should be noted that although the system frequency is the same at each bus at equilibrium, it can be different during the transient behaviour after a disturbance. This is a feature incorporated within our model.

To investigate decentralized control schemes for generation and controllable load based upon local measurements of the frequency alone, we close the loop in (3) by determining each of $p_{j}^{M}, d_{j}^{c}$, and $d_{j}^{u}$ as outputs from independent systems of the form in Section III with inputs given by the negative of the local frequency,

$$
\begin{aligned}
& \dot{x}^{M, j}=f^{M, j}\left(x^{M, j},-\omega_{j}\right), \\
& p_{j}^{M}=g^{M, j}\left(x^{M, j},-\omega_{j}\right), \\
& \dot{x}^{c, j}=f^{c, j}\left(x^{c, j},-\omega_{j}\right), \quad j \in G, \\
& d_{j}^{c}=g^{c, j}\left(x^{c, j},-\omega_{j}\right), \\
& \dot{x}^{u, j}=f^{u, j}\left(x^{u, j},-\omega_{j}\right), \quad \\
& d_{j}^{u}=g^{u, j}\left(x^{u, j},-\omega_{j}\right),
\end{aligned}
$$

For convenience in the notation we collect $^{5}$ the variables in (4) into the vectors $x^{M}=\left[x^{M, j}\right]_{j \in G}, x^{c}=\left[x^{c, j}\right]_{j \in N}$, and $x^{u}=\left[x^{u, j}\right]_{j \in N}$. These quantities represent the internal states of the dynamical systems ${ }^{6}$ used to update the desired outputs $p_{j}^{M}, d_{j}^{c}$, and $d_{j}^{u}$. The variables $p_{j}^{M}$ and $d_{j}^{c}$ are controllable, so we have freedom in our analysis to design certain properties of the dynamics in (4a) and (4b). By contrast, $d_{j}^{u}$ represents uncontrollable load and the dynamics in (4c) are thus fixed. Note that the systems in (4) can be heterogeneous and of arbitrary dimension.

Throughout the paper we aim to characterize broad classes of dynamics associated with generation and demand, so that stability and optimality can be guaranteed for the equilibrium points of the overall interconnected system (3)-(4).

\footnotetext{
${ }^{3} \mathrm{~A}$ nominal value of a variable is defined as its value at an equilibrium of (3) with frequency equal to the nominal value of $50 \mathrm{~Hz}$ (or $60 \mathrm{~Hz}$ ).

${ }^{4}$ The quantities $\eta_{i j}$ represent the phase differences between buses $i$ and $j$, given by $\theta_{i}-\theta_{j}$. The angles themselves must also satisfy $\dot{\theta}_{j}=\omega_{j}$ at all $j \in N$, however, we omit this equation in (3) since the power transfers $p$ are functions only of the phase differences.

${ }^{5}$ Each local variable (e.g. $x^{M, j}$ ) is a vector with multiple components.

${ }^{6}$ Note that since we allow general classes of dynamics for $p^{M}$ and $d^{u}$, system damping can be incorporated as part of these dynamics.
}

\section{B. Equilibrium analysis}

We now define the equilibria ${ }^{7}$ of the system (3)-(4).

Definition 1: The constants $\left(\eta^{*}, \omega^{*}, x^{M, *}, x^{c, *}, x^{u, *}\right)$ define an equilibrium of the system (3)-(4) if the following hold

$$
\begin{gathered}
0=\omega_{i}^{*}-\omega_{j}^{*},(i, j) \in E \\
0=-p_{j}^{L}+p_{j}^{M, *}-\left(d_{j}^{c, *}+d_{j}^{u, *}\right)-\sum_{k: j \rightarrow k} p_{j k}^{*}+\sum_{i: i \rightarrow j} p_{i j}^{*}, j \in G \\
0=-p_{j}^{L}-\left(d_{j}^{c, *}+d_{j}^{u, *}\right)-\sum_{k: j \rightarrow k} p_{j k}^{*}+\sum_{i: i \rightarrow j} p_{i j}^{*}, j \in L, \\
x^{M, j, *}=k_{x^{M, j}}\left(-\omega_{j}^{*}\right), j \in G, \\
x^{c, j, *}=k_{x^{c, j}}\left(-\omega_{j}^{*}\right), x^{u, j, *}=k_{x^{u, j}}\left(-\omega_{j}^{*}\right), j \in N
\end{gathered}
$$

where the quantities in (5b) and (5c) are given by

$$
\begin{gathered}
p_{i j}^{*}=B_{i j} \sin \eta_{i j}^{*}-p_{i j}^{n o m},(i, j) \in E, \\
p_{j}^{M, *}=k_{p_{j}^{M}}\left(-\omega_{j}^{*}\right), j \in G, \\
d_{j}^{c, *}=k_{d_{j}^{c}}\left(-\omega_{j}^{*}\right), d_{j}^{u, *}=k_{d_{j}^{u}}\left(-\omega_{j}^{*}\right), j \in N .
\end{gathered}
$$

We call (5) the equilibrium conditions for the system (3)-(4).

Throughout the remainder of the paper we suppose that there exists some equilibrium of (3)-(4) as defined in Definition 1 . We let $\left(\eta^{*}, \omega^{*}, x^{M, *}, x^{c, *}, x^{u, *}\right)$ denote any such equilibrium and use $\left(p^{*}, p^{M, *}, d^{c, *}, d^{u, *}\right)$ to represent the corresponding quantities defined in $(5 \mathrm{f})-(5 \mathrm{~h})$. We now impose a security constraint on the equilibrium power flows generated (see e.g. [23]).

Assumption 1: $\left|\eta_{i j}^{*}\right|<\frac{\pi}{2}$ for all $(i, j) \in E$.

Note that the static input-output characteristic maps $k_{p_{j}^{M}}, k_{d_{j}^{c}}$, and $k_{d_{j}^{u}}$ relating power generation/demand with frequency, as defined in (2), completely characterize the effect of the dynamics (4) on the behaviour of the power system (3) at equilibrium. In our analysis, we will consider a class of dynamics within (4) for which any such equilibrium point is asymptotically stable. Within this class, we then consider appropriate conditions on these characteristic maps such that the values of the variables defined in $(5 \mathrm{~g})-(5 \mathrm{~h})$ are optimal for an appropriately constructed network optimization problem.

\section{Combined passive dynamics from generation and load}

In terms of the outputs in (4), we define the net supply variables

$$
\begin{gathered}
s_{j}^{G}=p_{j}^{M}-\left(d_{j}^{c}+d_{j}^{u}\right), j \in G, \\
s_{j}^{L}=-\left(d_{j}^{c}+d_{j}^{u}\right), j \in L .
\end{gathered}
$$

Correspondingly, their values at equilibrium can be written as $s_{j}^{G, *}=p_{j}^{M, *}-\left(d_{j}^{c, *}+d_{j}^{u, *}\right)$ and $s_{j}^{L, *}=-\left(d_{j}^{c, *}+d_{j}^{u, *}\right)$.

The variables defined in (6) evolve according to the dynamics in (4). Consequently, $s_{j}^{G}$ and $s_{j}^{L}$ can be viewed as outputs from these combined dynamical systems with inputs $-\omega_{j}$.

\footnotetext{
${ }^{7}$ The interconnected system (3)-(4) could in general have multiple equilibria. It should be noted that the assumption in section III of having a unique equilibrium point when the input is constant is a condition on the individual subsystems representing loads and generation and does not preclude their interconnection from having multiple equilibrium points.
} 
We now introduce a notion of passivity for systems of the form (1), which we will use for the dynamics of the supply variables defined in (6) to prove our main stability results.

Definition 2: The system (1) is said to be locally input strictly passive about the constant input values $\bar{u}$ and the constant state values $\bar{x}$ if there exist open neighbourhoods $U$ of $\bar{u}$ and $X$ of $\bar{x}$ and a continuously differentiable, positive semidefinite function $V(x)$ (the storage function) such that, for all $u \in U$ and all $x \in X, \dot{V}(x) \leq(u-\bar{u})^{T}(y-\bar{y})-\phi(u-\bar{u})$, where $\phi$ is a positive definite function and $\bar{y}=k_{y}(\bar{u})$. If the regions $U$ and $X$ are the whole of $\mathbb{R}$ and $\mathbb{R}^{m}$ respectively, we say that system (1) is globally input strictly passive about the equilibrium point specified.

Remark 1: The storage function can be interpreted as a form of internal energy of the system. The passivity property can easily be checked for static nonlinearities, and one of its important features is that for a general linear system it can be verified by means of computationally efficient methods. In particular, it follows from the KYP Lemma [24] that passivity of a linear system is equivalent to the feasibility of a linear matrix inequality (LMI), i.e. a computationally efficient convex optimization problem from which the storage function can also be constructed. Passivity can also be checked for linear systems from the positive realness of the corresponding transfer function, using the fact that positive realness is equivalent for stable systems to the frequency response function lying in the right half-plane. Various examples involving nonlinear and linear dynamics will be discussed in Section VI.

We suppose that the supply dynamics (6) at each bus satisfy the local passivity condition in Definition 2. This is a decentralized condition, since it involves only the local supply dynamics at each bus.

Assumption 2: Each of the systems defined in (4) with inputs $-\omega_{j}$ and outputs given by (6a) and (6b) respectively are locally input strictly passive about their equilibrium values $-\omega_{j}^{*}$ and $\left(x^{M, j, *}, x^{c, j, *}, x^{u, j, *}\right)$, in the sense described in Definition 2.

Remark 2: It should be noted that the passivity property is assumed without specifying the precise form of the systems, which permits the inclusion of a broad class of generation and load dynamics. Also the fact that passivity is assumed only for the net supply dynamics, rather than for the generation and load dynamics individually, can permit the analysis of systems incorporating dynamics that are not individually passive.

\section{Optimal supply and load control}

We aim to explore how the generated power and controllable loads may be adjusted to meet the step change $p^{L}$ in frequency-independent load in a way that minimizes the total cost that comes from the extra power generated and the disutility of loads. We now introduce an optimization problem, which we call the optimal supply and load control problem (OSLC), that can be used to achieve this goal.

Suppose that costs $C_{j}\left(p_{j}^{M}\right)$ and $C_{d j}\left(d_{j}^{c}\right)$ are incurred for deviations $p_{j}^{M}$ and $d_{j}^{c}$ in generation and controllable load respectively. Furthermore, some additional cost is incurred due to any change in frequency which alters the uncontrollable frequency-dependent demand. We represent this by an integral $\operatorname{cost}^{8}$ in terms of a function $h_{j}$ which is determined by the dynamics in $(4 c)$ as

$$
h_{j}(z)=k_{d_{j}^{u}}(-z) \text { for all } z \in \mathbb{R} .
$$

The total cost within OSLC then sums all the above costs, and the problem is to choose the vectors $p^{M}, d^{c}$, and $d^{u}$ that minimize this total cost and simultaneously achieve power balance, while satisfying physical saturation constraints.

$$
\begin{aligned}
& \text { OSLC }: \\
& \min _{p^{M}, d^{c}, d^{u}} \sum_{j \in G} C_{j}\left(p_{j}^{M}\right)+\sum_{j \in N}\left(C_{d j}\left(d_{j}^{c}\right)+\int_{0}^{d_{j}^{u}} h_{j}^{-1}(z) d z\right) \\
& \text { subject to } \sum_{j \in G} p_{j}^{M}=\sum_{j \in N}\left(d_{j}^{c}+d_{j}^{u}+p_{j}^{L}\right), \\
& p_{j}^{M, \min } \leq p_{j}^{M} \leq p_{j}^{M, \max }, \forall j \in G, \\
& d_{j}^{c, \text { min }} \leq d_{j}^{c} \leq d_{j}^{c, \max }, \forall j \in N,
\end{aligned}
$$

where $p_{j}^{M, \min }, p_{j}^{M, \max }, d_{j}^{c, \min }$, and $d_{j}^{c, \max }$ are the bounds for generation and controllable demand respectively at bus $j$. The equality constraint in (8) represents conservation of power, i.e. that all the frequency-independent load is matched by the total generation plus all the frequency-dependent load contributions.

Remark 3: The variables $p^{M}$ and $d^{c}$ within (8) represent the variables that can be directly controlled, while the variable $d^{u}$ can be controlled only indirectly by effecting changes in the system frequencies. Therefore, we aim to specify properties of the control dynamics in (4a)-(4b) that ensure that the quantities $p^{M}$ and $d^{c}$, along with the system frequencies, converge to values at which optimality in (8) can be guaranteed.

\section{E. Additional conditions}

To guarantee convergence and optimality, we will require additional conditions on the behaviour of the systems (3)-(4) and the structure of the optimization problem (8). The assumptions introduced are all of practical relevance, and we will see in Section VI that the framework considered encompasses a number of important examples from the literature. Within the second condition we denote $\omega^{G}=\left[\omega_{j}\right]_{j \in G}$ and $\omega^{L}=\left[\omega_{j}\right]_{j \in L}$.

Assumption 3: The storage functions in Assumption 2 have strict local minima at the points $\left(x^{M, j, *}, x^{c, j, *}, x^{u, j, *}\right)$ and $\left(x^{c, j, *}, x^{u, j, *}\right)$ respectively.

Remark 4: In practice, Assumption 3 is often trivially satisfied. For instance, if the vector fields in (4) are continuously differentiable, then by linearizing about equilibrium, the KYP Lemma generates a storage function satisfying Assumption 3 whenever the linearized system is controllable and observable.

Assumption 4: There exists an open neighbourhood $T$ of $\left(\eta^{*}, \omega^{G, *}, x^{M, *}, x^{c, *}, x^{u, *}\right)$ such that at any time instant $t, \omega^{L}(t)$ is uniquely determined by the system states $\left(\eta(t), \omega^{G}(t), x^{M}(t), x^{c}(t), x^{u}(t)\right) \in T$ and equations (3)-(4), and the map relating the system states to $\omega^{L}(t)$ is locally Lipschitz.

\footnotetext{
${ }^{8}$ We use this alternative representation for the cost, in order to express the cost incurred as a function of properties of the system dynamics, as for uncontrollable loads no design of control system dynamics is feasible.
} 
Remark 5: Assumption 4 is a technical assumption that is required in order for the system (3)-(4) to have a locally well-defined state space realization. This is needed in order to apply Lasalle's Theorem to analyze stability in the proof of Theorem 1 below.

Remark 6: Assumption 4 can often be verified by using the Implicit Function Theorem to generate decentralized algebraic conditions under which it is guaranteed to hold. For instance, Assumption 4 always holds if in (4) we have that for all $j \in L$ functions $g^{c, j}, g^{u, j}$ are continuously differentiable and $\frac{\partial g^{c, j}}{\partial \omega_{j}}+\frac{\partial g^{u, j}}{\partial \omega_{j}} \neq 0$ at the equilibrium point. If the functions $g^{c, j}$ and $g^{u, j}$ have no explicit dependence on $\omega_{j}$, satisfying $\sum_{i} \frac{\partial g^{c, j}}{\partial x_{i}^{c, j}} \frac{\partial f_{i}^{c, j}}{\partial \omega_{j}}+\sum_{i} \frac{\partial g^{u, j}}{\partial x_{i}^{u, j}} \frac{\partial f_{i}^{u, j}}{\partial \omega_{j}}>0$ at the equilibrium point is also sufficient. These conditions are invoked in Section VII.

Assumption 5: The cost functions $C_{j}$ and $C_{d j}$ are continuously differentiable and strictly convex. Moreover, the first derivative of $h_{j}^{-1}(z)$ is nonnegative for all $z \in \mathbb{R}$.

\section{MAIN RESUlTS}

In this section we state our main results, with the proofs of Theorems 1-4 provided in Appendix A. Our first result shows that the set of equilibria of the system (3)-(4) for which the assumptions stated are satisfied is asymptotically attracting, while our second result demonstrates sufficient conditions for equilibrium points to be optimal for the OSLC problem (8). Based on these results, we can guarantee convergence to optimality of all solutions starting in the vicinity of an equilibrium. Finally, we show that the inclusion of controllable demand in our model reduces steady state frequency deviation, thereby aiding in frequency control.

Theorem 1 (Stability): Suppose that Assumptions 1-4 are all satisfied. Then there exists an open neighbourhood $S$ of the equilibrium $\left(\eta^{*}, \omega^{G, *}, x^{M, *}, x^{c, *}, x^{u, *}\right)$ such that whenever the initial conditions $\left(\eta(0), \omega^{G}(0), x^{M}(0), x^{c}(0), x^{u}(0)\right) \in S$, then the solutions of the system (3)-(4) converge to an equilibrium as defined in Definition 1.

Remark 7: It will be seen within the proof of Theorem 1 that $\omega, x^{M}, x^{c}, x^{u}$ converge to $\omega^{*}, x^{M, *}, x^{c, *}, x^{u, *}$ respectively.

Theorem 2 (Optimality): Suppose that Assumption 5 is satisfied. If the control dynamics in (4a) and (4b) are chosen such that

$$
\begin{gathered}
k_{p_{j}^{M}}\left(\omega^{*}\right)=\left[\left(C_{j}^{\prime}\right)^{-1}\left(\omega^{*}\right)\right]_{p_{j}^{M, \min }}^{p_{j}^{M, \max }} \\
k_{d_{j}^{c}}\left(-\omega^{*}\right)=\left[\left(C_{d j}^{\prime}\right)^{-1}\left(\omega^{*}\right)\right]_{d_{j}^{c, \min }}^{d_{j, \max }}
\end{gathered}
$$

then the values $p^{M, *}, d^{c, *}$, and $d^{u, *}$ are optimal for the OSLC problem (8).

Theorem 3 (Convergence to optimality): Consider equilibria of (3)-(4) with respect to which Assumptions 1-5 are all satisfied. If the control dynamics in (4a) and (4b) are chosen such that (9) holds, then there exists an open neighbourhood of initial conditions about any such equilibrium such that the solutions of (3)-(4) are guaranteed to converge to a global minimum of the OSLC problem (8).

Remark 8: Theorem 3 states that if the system (3)-(4) starts sufficiently close to any of its equilibria with respect to which
Assumptions 1-5 are satisfied, then the system converges to an equilibrium point which is optimal for the OSLC problem (8). The fact that $p^{M}$ and $d^{c}$ represent controllable quantities means that we are free to design the dynamics in (4a) and (4b) in order that the conditions (9) are satisfied. Thus, knowledge of the cost functions in the optimization problem we want to solve explicitly determines classes of dynamics which are guaranteed to yield convergence to optimal solutions.

Theorem 4: Suppose that Assumption 5 is satisfied. If the control dynamics in (4a) and (4b) are chosen such that (9) holds, then the addition of controllable demand in primary control results in a drop in the steady state frequency deviation from its nominal value.

\section{DISCUSSION}

We now discuss various examples of generation and load dynamics that can fit within our framework.

As a first example, consider the model in [13], which investigates a linearized version of the system (3) coupled with the static nonlinearities $d_{j}^{c}=\left(C_{d j}^{\prime}\right)^{-1}\left(\omega_{j}\right)$ for the controllable demand, and with uncontrollable loads of the form $d_{j}^{u}=$ $D_{j} \omega_{j}$. The damping constants $D_{j}$ were assumed positive, the cost functions $C_{d j}$ were taken to be strictly convex, and the mechanical power injection $p^{M}$ was also assumed to be constant after a step change. It is easy to see that for such a system Assumptions 1-5 are all satisfied. Hence, this model can be analyzed in the framework introduced above, thus implying optimality and stability of the equilibrium points.

The present framework can also include systems in which the generated powers satisfy any first-order dynamics as in [15], since such schemes are passive about their equilibria for arbitrary gains. For higher-order schemes, however, the dynamics for $p^{M}$ are not necessarily passive, so some additional conditions are needed to ensure stability. A significant example of this can be seen in the second-order generation dynamics that are often considered in literature to model turbine-governor dynamics, e.g. [10, p. 382]. These can be described by

$$
\begin{aligned}
& \dot{\alpha}_{j}=-\frac{1}{\tau_{g, j}} \alpha_{j}+\frac{1}{\tau_{g, j}} p_{j}^{c}, \\
& \dot{p}_{j}^{M}=-\frac{1}{\tau_{b, j}} p_{j}^{M}+\frac{1}{\tau_{b, j}} \alpha_{j},
\end{aligned} \quad j \in G
$$

where $\alpha_{j}$ is the valve position of the turbine, the constants $\tau_{g, j}$ and $\tau_{b, j}$ represent lags in the dynamics of the governor and turbine respectively, and $p_{j}^{c}$ is a static function of frequency, corresponding to droop control. Consider the case where generator damping and uncontrollable loads are modeled by

$$
d_{j}^{u}=D_{j} \omega_{j}, \forall j \in N
$$

and there is no controllable demand. In [14], the condition

$$
\left|p_{j}^{c}\left(\omega_{j}\right)-p_{j}^{c}\left(\omega_{j}^{*}\right)\right| \leq K_{j}\left|\omega_{j}-\omega_{j}^{*}\right|, j \in G
$$

with $K_{j}<D_{j}$ was imposed. 
As shown in Corollary 1 below, under (12), the overall system relating $-\omega_{j}$ with $^{9} s_{j}^{G}=p_{j}^{M}-d_{j}^{u}$ becomes input strictly passive about the equilibrium point considered. This follows from a more general result which we now state describing the connection between the $\mathcal{L}_{2}$-gain of general generation dynamics and the passivity of the supply dynamics. The proofs of Proposition 1 and Corollary 1 can be found in Appendix A.

Proposition 1: Let equation (11) hold and consider any generation dynamics from $-\omega_{j}$ to $p_{j}^{M}$ of the form (4a). Consider also the variable $p_{j}^{D}=p_{j}^{M}+g_{j}\left(\omega_{j}\right)$, where $g_{j}\left(\omega_{j}\right)$ is any function that is nondecreasing with respect to $\omega_{j}$. Given any equilibrium, if the $\mathcal{L}_{2}$-gain from $\left(\omega_{j}-\omega_{j}^{*}\right)$ to $\left(p_{j}^{D}-p_{j}^{D, *}\right)$ is strictly less than $D_{j}$, then the system with input $-\omega_{j}$ and output $s_{j}^{G}=p_{j}^{M}-d_{j}^{u}$ is globally input strictly passive about the equilibrium considered.

Remark 9: It should be noted that the proposition holds for dynamical systems from $\omega$ to $p^{M}$ of any order (not just second order) and the gain condition specified can be verified for broad classes of nonlinear systems. For example, if the system from $\left(p_{j}^{c}-p_{j}^{c, *}\right)$ to $\left(p_{j}^{M}-p_{j}^{M, *}\right)$ is linear with transfer function $G(s)$, with a nonlinearity at its input that satisfies (12), then it can be shown [22] that the $\mathcal{L}_{2}$-gain condition in the proposition is satisfied if $\sup _{\omega}|G(j \omega)| \leq 1$, by choosing $g_{j}\left(\omega_{j}\right)=0$. Less conservative conditions can also be deduced by choosing a nonzero $g_{j}$, as it will be shown in the proof of Corollary 1 below.

Remark 10: It should also be noted that the passivity property in Proposition 1 (and also in Lemma 1 and Corollary 1 below) holds globally about the equilibrium point considered, i.e., for all values of the inputs and states of the system specified (see Definition 2).

It is easy to show that for the dynamics (10), (11), (12), the condition in Proposition 1 is satisfied, and therefore the passivity property is satisfied (stated in Lemma 1 in the appendix) thus recovering the stability condition in [14]. In fact, it can be shown that Proposition 1 allows also to relax the gain condition in (12) to a less conservative condition as stated in the Corollary below.

Corollary 1: Consider the generation dynamics in (10) and let equation (11) and ${ }^{10}$ (9a) hold. Then, for any equilibrium where (12) holds with $K_{j}<1.53 D_{j}$, the system with input $-\omega_{j}$ and output $s_{j}^{G}=p_{j}^{M}-d_{j}^{u}$ is globally input strictly passive about this equilibrium.

Remark 11: It should be noted that Corollary 1 allows to deduce asymptotic stability with a gain condition that is less restrictive than the condition $K_{j}<D_{j}$ in [14] and does not make use of any linearisation of the system model.

Furthermore, our framework can allow us to deduce asymptotic stability under weaker conditions than those in (12),

\footnotetext{
${ }^{9}$ Note that this example could also include passive controllable demand $d_{j}^{c}\left(-\omega_{j}\right)$, since showing input strict passivity about equilibrium of the system with input $-\omega_{j}$ and output $s_{j}^{G}=p_{j}^{M}-d_{j}^{u}$ is sufficient to ensure also that the system with the same input and output $s_{j}^{G}=p_{j}^{M}-d_{j}^{u}-d_{j}^{c}$ is input strictly passive.

${ }^{10}$ Note that in $(9 \mathrm{a}) C_{j}($.$) is allowed to be any strictly convex function,$ which implies that $p_{c}($.$) is a non decreasing function. It can be shown that$ Corollary 1 still holds if this is relaxed to the mild condition that the deviations of $p_{c}(-\omega)$ from the equilibrium point have the same sign as those of $-\omega$.
}

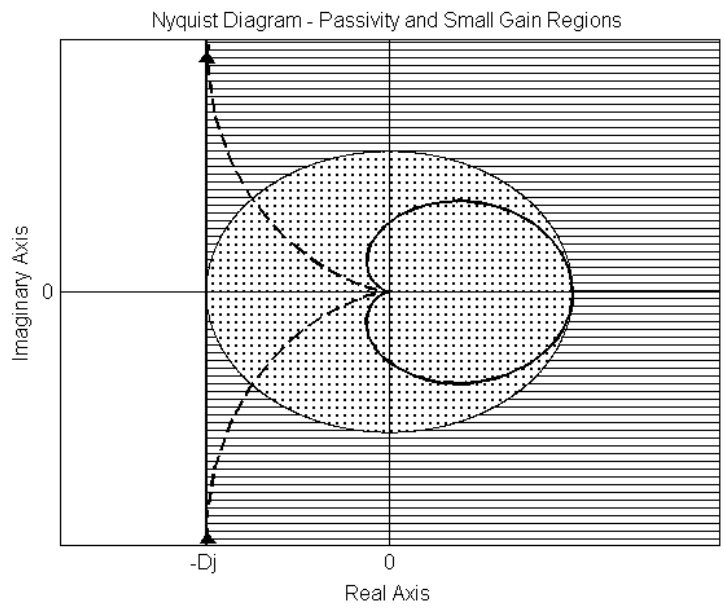

Fig. 2. Nyquist plot for the transfer function relating $\hat{\tilde{p}}_{j}^{M}$ with $-\hat{\tilde{\omega}}_{j}$ when a linearization of (10) about equilibrium is considered. A transfer function with a Nyquist plot within the circle satisfies the gain condition (12). Our approach allows the Nyquist plot to extend within the entire striped region.

Corollary 1 and Proposition 1, when linear generation dynamics are considered. To see this, we consider a linearization of the system (10) about equilibrium and let $\tilde{q}$ denote the deviation of any quantity $q$ from its equilibrium value $q^{*}$. Expressing $\tilde{p}_{j}^{M}$ in the Laplace domain gives $\hat{\tilde{p}}_{j}^{M}=$ $\frac{1}{\left(\tau_{g, j} s+1\right)\left(\tau_{b, j} s+1\right)} \hat{\tilde{p}}_{j}^{c}$. Therefore,

$$
\begin{aligned}
\hat{\tilde{s}}_{j}^{G}=\hat{\tilde{p}}_{j}^{M}-\hat{\tilde{d}}_{j}^{u} & =\frac{1}{\left(\tau_{g, j} s+1\right)\left(\tau_{b, j} s+1\right)} \hat{\tilde{p}}_{j}^{c}+D_{j}\left(-\hat{\tilde{\omega}}_{j}\right) \\
& =: H_{j}(s)\left[-\hat{\tilde{\omega}}_{j}\right], j \in G,
\end{aligned}
$$

where $H_{j}(s)$ denotes the transfer function relating $-\hat{\tilde{\omega}}_{j}$ and $\hat{\tilde{s}}_{j}^{G}$. Since the maximum gain of the transfer function from $\tilde{p}_{j}^{c}$ to $\tilde{p}_{j}^{M}$ is 1 at $s=0$, the condition in (12) constrains the Nyquist diagram of $H_{j}$ to lie inside a ball with centre $\left(D_{j}, 0\right)$ and radius $K_{j}<D_{j}$. This is contained strictly within the right half-plane, implying the required passivity condition in Assumption 2. For instance, the Nyquist plot from input $-\hat{\tilde{\omega}}_{j}$ to output $\hat{\tilde{p}}_{j}^{M}$ can be as shown by the solid line in Fig. 2. However, according to our analysis any dynamics for the command signal can be permitted provided that the supply dynamics in (13) remain input-strictly passive. This can permit any frequency response within the striped region in Fig. 2, for example allowing the larger Nyquist locus shown with a dashed line. In fact, under the reasonable assumption that $p_{j}^{c}$ has the same sign as $-\omega_{j}$ (i.e. negative feedback is used), it can easily be verified that the transfer function from $\tilde{p}_{j}^{c}$ to $\tilde{p}_{j}^{M}$ given by $T_{j}(s)=\frac{1}{\left(\tau_{g, j} s+1\right)\left(\tau_{b, j} s+1\right)}$ has a minimum real value

$$
\Re\left(T_{j}\left(j \omega_{j}^{M}\right)\right)=\frac{-\tau_{g, j} \tau_{b, j}}{\left(\tau_{g, j}+\tau_{b, j}\right)^{2}+2\left(\tau_{g, j}+\tau_{b, j}\right) \sqrt{\tau_{g, j} \tau_{b, j}}}
$$

at frequency $\omega_{j}^{M}=\sqrt{\frac{\left(\tau_{g, j}+\tau_{b, j}\right)+\sqrt{\tau_{b, j} \tau_{g, j}}}{\left(\tau_{b, j} \tau_{g, j}\right)^{3 / 2}}}$. Thus, the required passivity property will be maintained provided $K_{j}$ multiplied by the quantity in (14) is strictly greater than $-D_{j}$. Analysis of (14) shows that the maximum allowable value for $K_{j}$ is always at least $8 D_{j}$ (obtained at $\frac{\tau_{b, j}}{\tau_{g, j}}=1$ ) and tends to infinity as $\frac{\tau_{b, j}}{\tau_{g, j}} \rightarrow 0$ (which corresponds to a first order system). This shows that the stability guarantees can be preserved under 
significantly larger gains $K_{j}$ than the damping coefficients $D_{j}$. Therefore, our approach allows for a less conservative local stability condition for equilibrium points where a linearization is feasible, while also allowing to consider a wider class of generation dynamics.

Note, however, that the use of stability conditions derived from the more conservative $\mathcal{L}_{2}$-gain condition in Proposition 1 would generally be expected to yield better robustness properties. Such trade-offs between gain and stability margin need to be taken into account in the design of control systems.

In order to to further illustrate the generality of our approach we consider below a 5th order model for the turbine/governor dynamics which is a more realistic model used by the power system toolbox [25]. This leads to the transfer function below relating the mechanical power $p_{j}^{M}$ with the negative frequency deviation $-\omega_{j}$

$$
G_{j}(s)=K_{j} \frac{1}{\left(1+s T_{s, j}\right)} \frac{\left(1+s T_{3, j}\right)}{\left(1+s T_{c, j}\right)} \frac{\left(1+s T_{4, j}\right)}{\left(1+s T_{5, j}\right)}
$$

where $K_{j}$ and $T_{s, j}, T_{3, j}, T_{c, j}, T_{4, j}, T_{5, j}$ are the droop coefficient and time-constants respectively. Realistic values for these variables are provided by the toolbox data files for the turbine governor systems within the Northeast Power Coordinating Council (NPCC) network ${ }^{11}$. The passivity property required by our theory is satisfied if the Nyquist plot of $G_{j}(s)+D_{j}$, where $D_{j}$ is the generator damping, is in the right half-plane. Figure 3 shows such plots for various buses with turbine governor systems in the NPCC network where this property is satisfied. In particular, 20 out of the 22 NPCC buses with generators with turbine governor systems satisfy the passivity property (for the other 2 buses the condition is satisfied when the damping coefficients are increased by $37 \%$ and $28 \%$ respectively). Hence the passivity property is satisfied by many existing droop control implementations and is therefore not restrictive. Note that the significance of this property is that it is a decentralized condition, and it therefore provides a plug and play capability within the network when satisfied by all buses.

Furthermore, in order to investigate the condition in Proposition 1, we have included in Table I the values of the droop coefficients $K_{j}$ and damping coefficients $D_{j}$ of these generators. It can be seen that the droop coefficients are in most cases significantly larger than the corresponding damping coefficients. Therefore the condition $K_{j}<D_{j}$ that follows from Proposition 1 is not satisfied ${ }^{12}$ and large reductions in the feedback gain are needed to enforce it.

A further class of droop control schemes used in practice incorporates a deadband which prevents unneeded adjustments for small variations in frequency about nominal. For such systems, a minimum frequency deviation $\omega_{j}^{\text {min }}$ is required to trigger a frequency-dependent deviation and devices reach their physical limits at a higher frequency deviation $\omega_{j}^{\max }$.

\footnotetext{
${ }^{11}$ The data can be found in the Power System Toolbox file datanp 48 that provides parameter values for the NPCC 48 machine system.

${ }^{12}$ Buses 23 and 54 have two generators connected to them in the NPCC model. The table shows the parameters associated with one of the generators at each bus, but it should be noted that Proposition 1 is also not satisfied when the aggregate bus dynamics are taken into account.
}

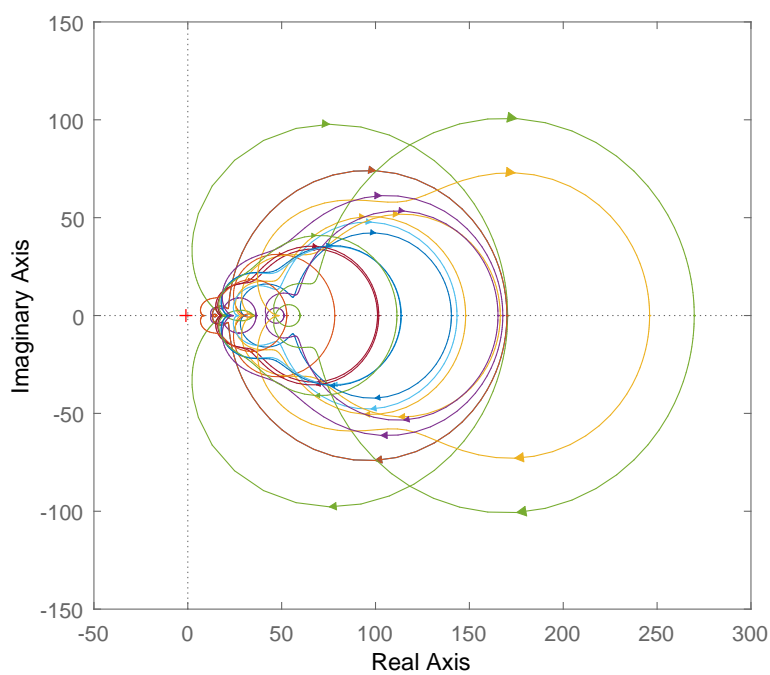

Fig. 3. Nyquist plots of the transfer functions relating $s_{j}$ with $-\omega_{j}$ for buses with turbine governor systems in the NPCC network where the passivity property is satisfied.

\begin{tabular}{|c|c|c||c|c|c|}
\hline Bus & $\begin{array}{c}\text { Damping } \\
\text { Coeff. } \\
\text { (p.u.) }\end{array}$ & $\begin{array}{c}\text { Droop } \\
\text { Coeff. } \\
\text { (p.u.) }\end{array}$ & Bus & $\begin{array}{c}\text { Damping } \\
\text { Coeff. } \\
\text { (p.u.) }\end{array}$ & $\begin{array}{c}\text { Droop } \\
\text { Coeff. } \\
\text { (p.u.) }\end{array}$ \\
\hline 21 & 34.8 & 43.5 & 50 & 34.44 & 132 \\
22 & 28.6 & 119.4 & 51 & 21.46 & 76.8 \\
23 & 7.34 & 25.8 & 54 & 51.24 & 180 \\
24 & 34.8 & 135.6 & 55 & 59.78 & 210 \\
25 & 26.4 & 117 & 56 & 21.94 & 91.8 \\
26 & 3.42 & 165.6 & 57 & 17.71 & 84 \\
27 & 24.3 & 123.4 & 60 & 21.74 & 91.8 \\
36 & 30.3 & 110.1 & 61 & 8.32 & 44.4 \\
42 & 18.86 & 82.2 & 79 & 48 & 198 \\
47 & 15.17 & 154.8 & 80 & 23.8 & 144 \\
48 & 15.17 & 154.8 & 82 & 19.6 & 91.8 \\
\hline
\end{tabular}

TABLE I. Droop and damping coefficients for generators in the NPCC Network.

While the stability of these systems can be shown by Theorem 1, provided Assumptions 1-4 hold, Theorem 2 cannot be applied, since the deadband leads to cost functions that are not continuously differentiable and hence do not satisfy Assumption 5. However, it can be shown by means of subgradient methods (see the extended version [26]) that Assumption 5 can be relaxed to include non-continuously differentiable cost functions, thereby permitting the application of our results to analyze systems with such dynamics.

Finally, it should be noted that our analysis could be relevant to analyze stability and optimality when changes in either generation or demand occur. For the case of persistent disturbances, due to e.g. renewable generation, our framework could be relevant when the timescale of those disturbances is slower than the timescale needed for the primary frequency control dynamics to reach equilibrium, i.e. typically longer than a few seconds. For faster disturbances, our analysis is also significant in the sense that lack of stability guarantees, e.g. due to insufficient damping in the system, is likely to lead to an amplification of these fluctuations within the network. Also a very conservative design (e.g. due to very small droop control gains), will lead to a system that is very slow in its 
response to disturbances.

\section{Simulations ON IEEE BUS SYSTEMS}

\section{A. Simulation on the IEEE 68-bus transmission system}

In this section we illustrate our results through applications on the IEEE New York / New England 68-bus interconnection system [27], simulated using the Power System Toolbox [25]. This is more detailed and realistic than our analytical model, including line resistances, a DC12 exciter model, power system stabilizer (PSS), and a subtransient reactance generator model. A similar model without PSS is used for comparison ${ }^{13}$.

The test system contains 52 load buses serving different types of loads including constant active and reactive loads. The overall system has a total real power of $16.41 \mathrm{GW}$. For our simulation, we added three loads on units 2, 9, and 17, each having a step increase of magnitude 1 p.u. (base 100MVA) at $t=1$ second. We allow controllable demand on 34 load buses with loads controlled every 10ms. The disutility function for the aggregate load at each bus is $d_{j}^{c}$ is $C_{d j}\left(d_{j}^{c}\right)=\frac{1}{2} \alpha_{j}\left(d_{j}^{c}\right)^{2}$. Cost coefficients $\alpha_{j}$ were selected such that the power allocated between total generation and controllable demand would be roughly equal, as suggested in [28]. The selected values were ${ }^{14} \alpha_{j}=4$ for load buses 1-10 and $\alpha_{j}=2$ for the rest.

Consider the static and dynamic ${ }^{15}$ control schemes given by ${ }^{16} d_{j}^{c}=\left(C_{d j}^{\prime}\right)^{-1}\left(\omega_{j}\right), j \in N$, and $\dot{d}_{j}^{c}=-\left(C_{j}^{\prime}\left(d_{j}^{c}\right)-\right.$ $\left.\omega_{j}\right), j \in N$. We refer to the resulting dynamics as Static OSLC and Dynamic OSLC respectively. We investigate the behaviour in the following six cases: (i) no OSLC, no PSS, (ii) Static OSLC, no PSS, (iii) Dynamic OSLC, no PSS, (iv) no OSLC, with PSS, (v) Static OSLC, with PSS, (vi) Dynamic OSLC, with PSS. The pre-disturbance conditions of the simulations for the bus voltages, net injections and power transfers are given in Appendix B.

The frequency dynamics for bus 63 are shown in Fig. 4(a). From Fig. 4(a), we observe that whether or not PSS is used, the presence of OSLC results in a drop in steady state frequency deviations. Furthermore, we see that the overshoot is significantly less when OSLC is used. The responses for Static and Dynamic OSLC have no significant differences and converge to the same exact value at steady state. However, Dynamic OSLC appears to give a larger overshoot than Static OSLC. In all cases, the voltage deviation was less than 0.015 p.u., showing that the constant voltage assumption is reasonable. In Fig. 4(b) we also observe a higher power allocation at the load buses whose cost coefficients take the lower value $\alpha_{j}=2$ than at those with $\alpha_{j}=4$. This

\footnotetext{
${ }^{13}$ The details of the simulation models with or without PSS can be found in the Power System Toolbox data files data $16 \mathrm{~m}$ and data16em respectively.

${ }^{14}$ The values of $\alpha_{j}$ are given throughout the paper in units consistent with the frequency measured in $\mathrm{Hz}$ and power in per unit. Diving by 60 gives the value of $\alpha$ with the frequency also in per unit.

${ }^{15}$ The dynamic control scheme corresponds to cases where there is a lag in the response of the loads, or cases where low pass filtering is introduced in the control policy to avoid changes in the demand due to faster variations in grid frequency.

${ }^{16}$ Note that both of these are input strictly passive about the equilibria in the presense of arbitrarily small frequency damping, and both satisfy Assumption 4 (using respectively the conditions in Remark 6).
}

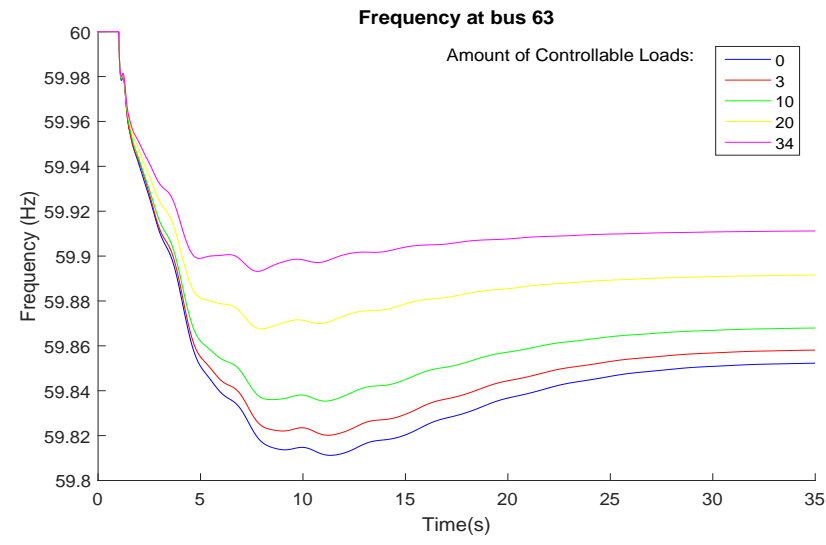

Fig. 5. Frequency at bus 63 with an increasing number of controllable loads.

demonstrates that the power allocation among controllable loads depends upon the loads' respective cost coefficients of demand response. This behaviour could be beneficial if a prescribed allocation were desirable, as then the load dynamics could be designed such that the cost coefficients chosen yield the desired allocation. Furthermore, as shown in Fig. 4(c), the marginal costs at each controlled load converge to the same value. This illustrates the optimality in division among loads, as equality of marginal costs is the optimality condition for (8) when the allocations do not saturate.

To study how the amount of controllable demand affects the grid frequency, we repeated the simulation on the system with PSS and static load control schemes, varying this time the number of controllable loads. The resulting time responses are shown on Figure 5. From there, it can be seen that an increase in the amount of controllable loads results to a reduced frequency deviation at all times. This therefore results in nadir and steady state values of frequency that are closer to the nominal frequency.

\section{B. Simulations on the IEEE 37-bus distribution system}

To illustrate the validity of our stability and optimality results on a lower voltage network, we simulated an appropriately modified balanced ${ }^{17}$ version of the IEEE 37-bus distribution system [29] using the Power System Toolbox. The test system is a feeder in California, with a $4.8 \mathrm{kV}$ operating voltage, fed by a big power system from one particular bus, which is modeled as an infinite bus. The system simulated is more realistic than our analytical model, and includes line resistances and reactive power as well as different types of balanced loads, as constant active and reactive power, constant impedance and constant current loads. The overall system has total real and reactive power of $2.52 \mathrm{MW}$ and $1.25 \mathrm{MVAr}$ respectively.

In order to examine the behaviour of controllable loads in a distribution system when a sudden change in the rest of the power grid occurs, we added a disturbance in the form of a step increase in load at the infinite bus at $t=1$ second, which results in a change $n$ the grid frequency. We allowed

\footnotetext{
${ }^{17}$ In particular, balanced loads were used and the simulation was carried out using the Power System Toolbox that uses single phase simulations.
} 

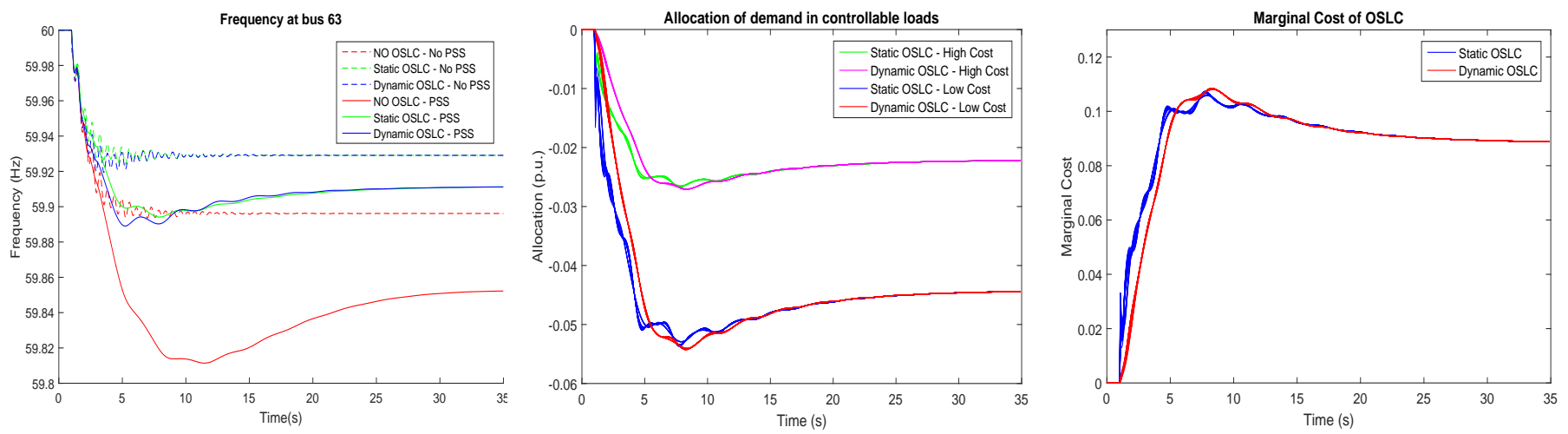

Fig. 4. (a) (left) Frequency at bus 63 in six cases: (i) no OSLC, no PSS, (ii) Static OSLC, no PSS, (iii) Dynamic OSLC, no PSS, (iv) no OSLC, with PSS, (v) Static OSLC, with PSS, (vi) Dynamic OSLC, with PSS, (b) (center) Power allocation among controllable loads with non-equal cost coefficients in two cases: (i) Static OSLC, (ii) Dynamic OSLC, (c) (right) Marginal costs $C_{d j}^{\prime}$ of controllable loads with non-equal cost coefficients, in two cases: (i) Static OSLC, (ii) Dynamic OSLC.

controllable loads on 24 buses with loads controlled every $10 \mathrm{~ms}$. Furthermore, we used the quadratic disutility function $C_{d j}\left(d_{j}^{c}\right)=\frac{1}{2} \alpha_{j}\left(d_{j}^{c}\right)^{2}$ with cost coefficient values of $a_{j}=4$ for load buses $712-714$ and $a_{j}=2$ for the rest. Fig. 6 shows the transient behaviour when Dynamic OSLC is used for the load control schemes (the response when Static OSLC is used is very similar).

The frequency at all buses is shown in Fig. 6(a) from where the stability of the system is demonstrated. The voltage deviation is larger than that in the IEEE 68-bus network simulation taking values up to 0.022 p.u., but still relatively small. In Fig. 6(b) we observe a higher power allocation at the load buses with the lower cost coefficient $\alpha_{j}=2$ than those with the higher cost coefficient $\alpha_{j}=4$. From Fig. 6(c) we can also see that, as in the IEEE 68-bus simulation, the marginal costs of all controllable loads converge to the same value.

\section{CONCLUSION}

We have considered the problem of designing distributed generation and demand control schemes for primary frequency regulation in power networks such that asymptotic stability is guaranteed while ensuring optimality of power allocation. We have presented a network passivity framework which provides a systematic method to show stability over a broad class of generation and load dynamics. Furthermore, we have derived steady state conditions for the generation and controllable demand control schemes that ensure that the power generated/consumed is the solution to an appropriately constructed network optimization problem, thus allowing fairness in power allocation to be guaranteed. In addition, under some minor assumptions, we have shown that the inclusion within the model of controllable demand has a positive effect also on secondary control, decreasing the steady state deviation in frequency. Simulations on the IEEE 68-bus and the IEEE 37bus systems verify our results. Interesting potential extensions to our analysis include the study of secondary frequency control, as well as the incorporation of voltage dynamics.

\section{APPENDIX A}

In this appendix we prove our main results, Theorems $1-3$. Proof of Theorem 1: We will use the dynamics in (3) together with the passivity conditions in Assumption 2 to define a Lyapunov function for system (3)-(4).

Firstly, we consider $V_{F}\left(\omega^{G}\right)=\frac{1}{2} \sum_{j \in G} M_{j}\left(\omega_{j}-\omega_{j}^{*}\right)^{2}$. The time-derivative along the trajectories of (3)-(4) is then $\dot{V}_{F}=$ $\sum_{j \in G}\left(\omega_{j}-\omega_{j}^{*}\right)\left(-p_{j}^{L}+s_{j}^{G}-\sum_{k: j \rightarrow k} p_{j k}+\sum_{i: i \rightarrow j} p_{i j}\right)+$ $\sum_{j \in L}\left(\omega_{j}-\omega_{j}^{*}\right)\left(-p_{j}^{L}+s_{j}^{L}-\sum_{k: j \rightarrow k} p_{j k}+\sum_{i: i \rightarrow j} p_{i j}\right)$, by substituting (3b) for $\dot{\omega}_{j}$ for $j \in G$ and adding the final term, which is equal to zero by (3c). Subtracting the product of $\left(\omega_{j}-\omega_{j}^{*}\right)$ with each term in $(5 b)$ and $(5 c)$, we get

$$
\begin{gathered}
\dot{V}_{F}=\sum_{j \in G}\left(\omega_{j}-\omega_{j}^{*}\right)\left(s_{j}^{G}-s_{j}^{G, *}\right)+\sum_{j \in L}\left(\omega_{j}-\omega_{j}^{*}\right)\left(s_{j}^{L}-s_{j}^{L, *}\right) \\
+\sum_{(i, j) \in E}\left(p_{i j}-p_{i j}^{*}\right)\left(\omega_{j}-\omega_{i}\right)
\end{gathered}
$$

using in the final term the equilibrium condition (5a).

Additionally, consider $V_{P}(\eta)=\sum_{(i, j) \in E} B_{i j} \int_{\eta_{i j}^{*}}^{\eta_{i j}}(\sin \phi-$ $\left.\sin \eta_{i j}^{*}\right) d \phi$. Using (3a) and (3d), the time-derivative equals

$$
\begin{aligned}
\dot{V}_{P} & =\sum_{(i, j) \in E} B_{i j}\left(\sin \eta_{i j}-\sin \eta_{i j}^{*}\right)\left(\omega_{i}-\omega_{j}\right) \\
& =\sum_{(i, j) \in E}\left(p_{i j}-p_{i j}^{*}\right)\left(\omega_{i}-\omega_{j}\right) .
\end{aligned}
$$

Now, from Assumption 2 and the definition of input strict passivity from Section III, it follows that for each $j \in G$, there exist open neighbourhoods $U_{j}^{G}$ of $\omega_{j}^{*}$ and $X_{j}^{G}$ of $\left(x^{M, j, *}, x^{c, j, *}, x^{u, j, *}\right)$ and a continuously differentiable, positive semidefinite function $V_{j}^{G}\left(x^{M, j}, x^{c, j}, x^{u, j}\right)$ such that

$$
\begin{aligned}
\dot{V}_{j}^{G} \leq\left(\left(-\omega_{j}\right)-\left(-\omega_{j}^{*}\right)\right)\left(s_{j}^{G}\right. & \left.-s_{j}^{G, *}\right) \\
& -\phi_{j}^{G}\left(\left(-\omega_{j}\right)-\left(-\omega_{j}^{*}\right)\right)
\end{aligned}
$$

for all $\omega_{j} \in U_{j}^{G}$ and all $\left(x^{M, j}, x^{c, j}, x^{u, j}\right) \in X_{j}^{G}$. Similarly, for each $j \in L$, there exist open neighbourhoods $U_{j}^{L}$ of $\omega_{j}^{*}$ and $X_{j}^{L}$ of $\left(x^{c, j, *}, x^{u, j, *}\right)$ and a continuously differentiable, positive semidefinite function $V_{j}^{L}\left(x^{c, j}, x^{u, j}\right)$ such that

$$
\begin{aligned}
\dot{V}_{j}^{L} \leq\left(\left(-\omega_{j}\right)-\left(-\omega_{j}^{*}\right)\right)\left(s_{j}^{L}\right. & \left.-s_{j}^{L, *}\right) \\
& -\phi_{j}^{L}\left(\left(-\omega_{j}\right)-\left(-\omega_{j}^{*}\right)\right)
\end{aligned}
$$



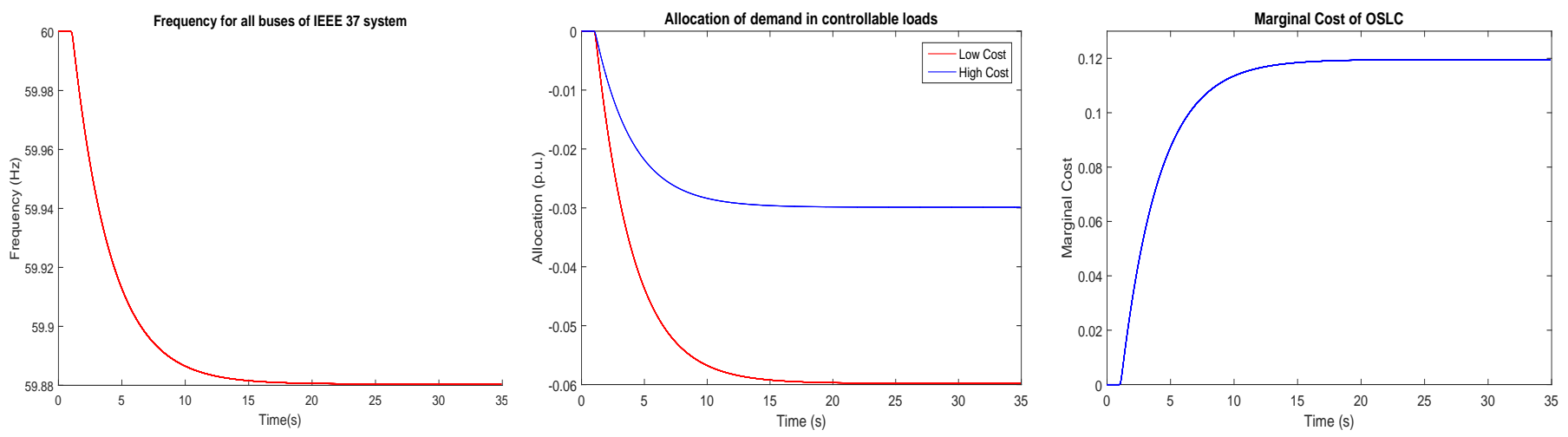

Fig. 6. (a) (left) Frequency at all buses for the IEEE 37-bus distribution system (b) (center) Power allocation among controllable loads with non-equal cost coefficients for Dynamic OSLC (c) (right) Marginal costs $C_{d j}^{\prime}$ of controllable loads with non-equal cost coefficients for Dynamic OSLC.

for all $\omega_{j} \in U_{j}^{L}$ and all $\left(x^{c, j}, x^{u, j}\right) \in X_{j}^{L}$. In (17) and (18), $\phi_{j}^{G}$ and $\phi_{j}^{L}$ are positive definite functions.

Based on the above, we define the function

$$
\begin{aligned}
& V\left(\eta, \omega^{G}, x^{M}, x^{c}, x^{u}\right)=V_{F}\left(\omega^{G}\right)+V_{P}(\eta) \\
& \quad+\sum_{j \in G} V_{j}^{G}\left(x^{M, j}, x^{c, j}, x^{u, j}\right)+\sum_{j \in L} V_{j}^{L}\left(x^{c, j}, x^{u, j}\right) .
\end{aligned}
$$

By (15) and (16), $\dot{V}=\sum_{j \in G}\left[\left(\omega_{j}-\omega_{j}^{*}\right)\left(s_{j}^{G}-s_{j}^{G, *}\right)+\right.$ $\left.\dot{V}_{j}^{G}\right]+\sum_{j \in L}\left[\left(\omega_{j}-\omega_{j}^{*}\right)\left(s_{j}^{L}-s_{j}^{L, *}\right)+\dot{V}_{j}^{L}\right]$. Using (17) and (18), it therefore holds that, whenever $\omega_{j} \in U_{j}^{G}$ and $\left(x^{M, j}, x^{c, j}, x^{u, j}\right) \in X_{j}^{G}$ for all $j \in G$ and $\omega_{j} \in U_{j}^{L}$ and $\left(x^{c, j}, x^{u, j}\right) \in X_{j}^{L}$ for all $j \in L$,

$$
\begin{aligned}
\dot{V} & \leq-\sum_{j \in G} \phi_{j}^{G}\left(\left(-\omega_{j}\right)-\left(-\omega_{j}^{*}\right)\right)-\sum_{j \in L} \phi_{j}^{L}\left(\left(-\omega_{j}\right)-\left(-\omega_{j}^{*}\right)\right) \\
& \leq 0 .
\end{aligned}
$$

Clearly $V_{F}$ has a strict global minimum at $\omega^{G, *}$, and $V_{j}^{G}, V_{j}^{L}$ have strict local minima at $\left(x^{M, j, *}, x^{c, j, *}, x^{u, j, *}\right)$, $\left(x^{c, j, *}, x^{u, j, *}\right)$ by Assumption 3. Additionally, Assumption 1 guarantees the existence of some neighbourhood of each $\eta_{i j}^{*}$ on which the respective integrand in the definition of $V_{P}$ is increasing. Since the integrand is zero at the lower limit, $\eta_{i j}^{*}$, this immediately implies that $V_{P}$ has a strict local minimum at $\eta^{*}$. Thus, $V$ has a strict local minimum at the point $Q^{*}:=\left(\eta^{*}, \omega^{G, *}, x^{M, *}, x^{c, *}, x^{u, *}\right)$. We now recall Assumption 4. This tells us that, provided $\left(\eta, \omega^{G}, x^{M}, x^{c}, x^{u}\right) \in T$, $\omega^{L}$ can be uniquely determined from these quantities. Therefore, the states of the differential equation system (3)-(4) within the region $T$ can be expressed as $\left(\eta, \omega^{G}, x^{M}, x^{c}, x^{u}\right)$. We can thus choose a neighbourhood in the coordinates $\left(\eta, \omega^{G}, x^{M}, x^{c}, x^{u}\right)$ about $Q^{*}$ on which the following all hold:

1) $Q^{*}$ is a strict minimum of $V$,

2) $\left(\eta, \omega^{G}, x^{M}, x^{c}, x^{u}\right) \in T$,

3) $\omega_{j} \in U_{j}^{G}$ and $\left(x^{M, j}, x^{c, j}, x^{u, j}\right) \in X_{j}^{G}$ for all $j \in G$ and $\omega_{j} \in U_{j}^{L}$ and $\left(x^{c, j}, x^{u, j}\right) \in X_{j}^{L}$ for all $j \in L^{18}$,

4) $x^{M, j}, x^{c, j}$, and $x^{u, j}$ all lie within their respective neighbourhoods $X_{0}$ as defined in Section III.

\footnotetext{
${ }^{18}$ This is possible because the requirement $\omega_{j} \in U_{j}^{L}$ for all $j \in L$ corresponds, by Assumption 4 and the continuity of (3)-(4), to requiring the states $\left(\eta, \omega^{G}, x^{M}, x^{c}, x^{u}\right)$ to lie in some open neighbourhood about $Q^{*}$.
}

Recalling now (19), within this neighbourhood $V$ is thus a nonincreasing function of all the system states and has a strict local minimum at $Q^{*}$. Consequently, the connected component of the level set $\left\{\left(\eta, \omega^{G}, x^{M}, x^{c}, x^{u}\right): V \leq \epsilon\right\}$ containing $Q^{*}$ is both compact and positively invariant with respect to (3)(4) for all sufficiently small $\epsilon>0$. Therefore, there exists a compact positively invariant set $\Xi$ for (3)-(4) containing $Q^{*}$.

Lasalle's Invariance Principle can now be applied with the function $V$ on the compact positively invariant set $\Xi$. This guarantees that all solutions of (3)-(4) with initial conditions $\left(\eta(0), \omega^{G}(0), x^{M}(0), x^{c}(0), x^{u}(0)\right) \in \Xi$ converge to the largest invariant set within $\Xi \cap\left\{\left(\eta, \omega^{G}, x^{M}, x^{c}, x^{u}\right): \dot{V}=0\right\}$. We now consider this invariant set. If $\dot{V}=0$ holds at a point within $\Xi$, then (19) holds with equality, whence by Assumption 2 we must have $\omega=\omega^{*}$. Moreover, on any invariant set on which $\omega=\omega^{*}$, the system equations (3) apply and give precisely the equilibrium conditions (5a), (5b), (5c), and (5f). Furthermore, if $\dot{V}=0$, it follows from (15), (16), (17), and (18) that all $\dot{V}_{j}^{G}=0$ and $\dot{V}_{j}^{L}=0$. But $\omega=\omega^{*}$ implies by the definitions in Section III that $\left(x^{M}, x^{c}, x^{u}\right)$ converge to the point $\left(x^{M, *}, x^{c, *}, x^{u, *}\right)$, at which Assumption 3 states that $V_{j}^{G}$ and $V_{j}^{L}$ take strict local minima. Therefore the values of $V_{j}^{G}$ and $V_{j}^{L}$ must decrease along all nontrivial trajectories within the invariant set, contradicting $\dot{V}_{j}^{G}=0$ and $\dot{V}_{j}^{L}=0$. Consequently, at all points of any invariant set within $\Xi \cap\left\{\left(\eta, \omega^{G}, x^{M}, x^{c}, x^{u}\right): \dot{V}=0\right\}$, we must also have $\left(x^{M}, x^{c}, x^{u}\right)=\left(x^{M, *}, x^{c, *}, x^{u, *}\right)$. Thus, the remaining equilibrium conditions (5d), (5e), (5g) and (5h) are also satisfied. Therefore, we conclude by Lasalle's Invariance Principle that all solutions of (3)-(4) with initial conditions $\left(\eta(0), \omega^{G}(0), x^{M}(0), x^{c}(0), x^{u}(0)\right) \in \Xi$ converge to the set of equilibria defined in Definition 1. Finally, choosing for $S$ any open neighbourhood of $Q^{*}$ within $\Xi$ completes the proof.

Remark 12: It should be noted that in the proof we do not exploit the fact that $\eta_{i j}=\theta_{i}-\theta_{j}$. It can be shown that by making use of this property the equilibrium value $\eta^{*}$ is unique for a given equilibrium frequency $\omega^{*}$.

Proof of Theorem 2: Due to Assumption 5, $C_{j}^{\prime}$ and $C_{d j}^{\prime}$ are strictly increasing and hence invertible. Therefore all variables in (9) with $\bar{u}=-\omega_{j}^{*}$ are well-defined. Furthermore, Assumption 5 also ensures that the OSLC problem (8) is a convex optimization problem with a continuously differentiable cost 
function. Thus, a point $\left(\bar{p}^{M}, \bar{d}^{c}, \bar{d}^{u}\right)$ is a global minimum for (8) if and only if it satisfies the KKT conditions [30]

$$
\begin{gathered}
C_{j}^{\prime}\left(\bar{p}_{j}^{M}\right)=-\nu-\lambda_{j}^{+}+\lambda_{j}^{-}, j \in G \\
C_{d j}^{\prime}\left(\bar{d}_{j}^{c}\right)=\nu-\mu_{j}^{+}+\mu_{j}^{-}, j \in N \\
h_{j}^{-1}\left(\bar{d}_{j}^{u}\right)=\nu, j \in N \\
\sum_{j \in G} \bar{p}_{j}^{M}=\sum_{j \in N}\left(\bar{d}_{j}^{c}+\bar{d}_{j}^{u}+p_{j}^{L}\right) \\
p_{j}^{M, m i n} \leq \bar{p}_{j}^{M} \leq p_{j}^{M, \max }, j \in G \\
d_{j}^{c, \text { min }} \leq \bar{d}_{j}^{c} \leq d_{j}^{c, m a x}, j \in N \\
\lambda_{j}^{+}\left(\bar{p}_{j}^{M}-p_{j}^{M, \max }\right)=0, \lambda_{j}^{-}\left(\bar{p}_{j}^{M}-p_{j}^{M, \min }\right)=0, j \in G, \\
\mu^{+}\left(\bar{d}_{j}^{c}-d_{j}^{c, \max }\right)=0, \mu^{-}\left(\bar{d}_{j}^{c}-d_{j}^{c, \min }\right)=0, j \in N
\end{gathered}
$$

for some constants $\nu \in \mathbb{R}$ and $\lambda_{j}^{+}, \lambda_{j}^{-}, \mu_{j}^{+}, \mu_{j}^{-} \geq 0$. We will now show that these conditions are satisfied by the equilibrium values $\left(\bar{p}^{M}, \bar{d}^{c}, \bar{d}^{u}\right)=\left(p^{M, *}, d^{c, *}, d^{u, *}\right)$ defined by equations $(5 \mathrm{~g})$ and $(5 \mathrm{~h})$.

Since $C_{j}^{\prime}$ and $C_{d j}^{\prime}$ are strictly increasing, we can uniquely define $\omega_{j}^{M, \max }:=-C_{j}^{\prime}\left(p_{j}^{M, \max }\right), \omega_{j}^{M, \min }:=-C_{j}^{\prime}\left(p_{j}^{M, \min }\right)$, $\omega_{j}^{c, \max }:=C_{d j}^{\prime}\left(d_{j}^{c, \max }\right)$, and $\omega_{j}^{c, \min }:=C_{d j}^{\prime}\left(d_{j}^{c, \text { min }}\right)$. Letting $\omega_{0}^{*}$ denote the common value of all $\omega_{j}^{*}$ due to $(5 a)$, we can, in terms of these quantities, define the nonnegative constants

$$
\begin{aligned}
\lambda_{j}^{+} & :=\left(\omega_{j}^{M, \max }-\omega_{0}^{*}\right) \mathbb{1}_{\left\{q: q \leq \omega_{j}^{M, \max }\right\}}\left(\omega_{0}^{*}\right), \\
\lambda_{j}^{-} & :=\left(\omega_{0}^{*}-\omega_{j}^{M, \min }\right) \mathbb{1}_{\left\{q: q \geq \omega_{j}^{M, \min \}}\right\}}\left(\omega_{0}^{*}\right), \\
\mu_{j}^{+} & :=\left(\omega_{0}^{*}-\omega_{j}^{c, \max }\right) \mathbb{1}_{\left\{q: q \geq \omega_{j}^{c, \max }\right\}}\left(\omega_{0}^{*}\right), \\
\mu_{j}^{-} & :=\left(\omega_{j}^{c, \min }-\omega_{0}^{*}\right) \mathbb{1}_{\left\{q: q \leq \omega_{j}^{c, \min }\right\}}\left(\omega_{0}^{*}\right) .
\end{aligned}
$$

Then, since $\left(C_{j}^{\prime}\right)^{-1}\left(-\omega_{0}^{*}\right) \geq p_{j}^{M, \max } \Leftrightarrow \omega_{0}^{*} \leq \omega_{j}^{M, \max }$, $\left(C_{j}^{\prime}\right)^{-1}\left(-\omega_{0}^{*}\right) \leq p_{j}^{M, \min } \Leftrightarrow \omega_{0}^{*} \geq \omega_{j}^{M, \min },\left(C_{d j}^{\prime}\right)^{-1}\left(\omega_{0}^{*}\right) \geq$ $d_{j}^{c, \max } \Leftrightarrow \omega_{0}^{*} \geq \omega_{j}^{c, \max }$, and $\left(C_{d j}^{\prime}\right)^{-1}\left(\omega_{0}^{*}\right) \leq d_{j}^{c, \text { min }} \Leftrightarrow \omega_{0}^{*} \leq$ $\omega_{j}^{c, m i n}$, it follows by (5g), (5h) and (9) that the complementary slackness conditions $(20 \mathrm{~g})$ and $(20 \mathrm{~h})$ are satisfied.

Now define $\nu=\omega_{0}^{*}$. Then $\left(C_{j}^{\prime}\right)^{-1}\left(-\nu-\lambda_{j}^{+}+\lambda_{j}^{-}\right)=$ $\left(C_{j}^{\prime}\right)^{-1}\left(\left[-\omega_{0}^{*}\right]_{-\omega_{j}^{M, \min }}^{-\omega_{j}^{M, \max }}\right)=\left[\left(C_{j}^{\prime}\right)^{-1}\left(-\omega_{0}^{*}\right)\right]_{p_{j}^{M, \min }}^{p_{M}^{M, \max }}=p_{j}^{M, *}$, by the above definitions and equations (5g) and (9). Thus, the optimality condition (20a) holds. Analogously, $\left(C_{d j}^{\prime}\right)^{-1}(\nu-$ $\left.\mu^{+}+\mu^{-}\right)=\left(C_{d j}^{\prime}\right)^{-1}\left(\left[\omega_{0}^{*}\right]_{\omega_{j}^{c, \text { min }}}^{\omega_{c, \text { max }}^{c}}\right)=\left[\left(C_{d j}^{\prime}\right)^{-1}\left(\omega_{0}^{*}\right)\right]_{d_{j}^{c, \text { min }}}^{d_{j}^{c, \text { max }}}=$ $d_{j}^{c, *}$, by (5h) and (9), satisfying (20b). Additionally, (20c) holds as $h_{j}(\nu)=d_{j}^{u}$ follows from (5h) and (7).

Furthermore, summing the equilibrium conditions $(5 \mathrm{~b})$ over all $j \in G$ and (5c) over all $j \in L$ shows that (20d) holds. Finally, the saturation constraints in (9) verify (20e) and (20f).

Thus, the values $\left(\bar{p}^{M}, \bar{d}^{c}, \bar{d}^{u}\right)=\left(p^{M, *}, d^{c, *}, d^{u, *}\right)$ satisfy the KKT conditions (20). Therefore, the equilibrium values $p^{M, *}, d^{c, *}$, and $d^{u, *}$ define a global minimum for (8).

Proof of Theorem 3: If Assumptions 1-5 all hold and (9) is true, then all of the assumptions in both Theorems 1 and 2 are satisfied, and thus the result follows.

Proof of Theorem 4: Recalling the proof of Theorem 2, we know from (20d) and the equalities (9) that at any equilibrium of (3)-(4) the power balance equation

$$
\sum_{j \in G}\left(C_{j}^{\prime}\right)^{-1}\left(\omega_{0}^{*}\right)+\sum_{j \in N}\left(\left(C_{d j}^{\prime}\right)^{-1}\left(\omega_{0}^{*}\right)+h_{j}\left(\omega_{0}^{*}\right)\right)=-\sum_{j \in N} p_{j}^{L}
$$

is satisfied, where $\omega_{0}^{*}$ denotes the common steady state value of frequency due to $(5 \mathrm{a})$. Now note that, because the nominal frequency defines an equilibrium frequency prior to the step change in load and all quantities in (3) denote deviations from their respective values at this nominal equilibrium, the equalities (7) and (9) imply that each term on the lefthand side in (21) must take the value zero at $\omega_{0}^{*}=0$. Furthermore, Assumption 5 implies that the terms $\left(C_{j}^{\prime}\right)^{-1}\left(\omega_{0}^{*}\right)$ and $\left(C_{d j}^{\prime}\right)^{-1}\left(\omega_{0}^{*}\right)$ are all strictly increasing in $\omega_{0}^{*}$, while each term $h_{j}\left(\omega_{0}^{*}\right)$ is nondecreasing in $\omega_{0}^{*}$. Thus both the added term due to load control and the entire left-hand side in (21) have the same sign as $\omega_{0}^{*}$ and are strictly increasing in $\omega_{0}^{*}$. It follows that the presence of this load control term results in a decrease in the value of $\omega_{0}^{*}$, the steady state frequency deviation from its nominal value.

Proof of Proposition 1: The $\mathcal{L}_{2}$-gain condition implies ${ }^{19}$

$$
\sqrt{\int_{0}^{t_{1}}\left(\tilde{p}_{j}^{D}\right)^{2} d t} \leq K_{j} \sqrt{\int_{0}^{t_{1}} \tilde{\omega}_{j}^{2}(t) d t} .
$$

where $K_{j}<D_{j}$ and $t_{1}$ is any positive constant. Then, input strict passivity can be shown as follows.

$$
\begin{aligned}
\int_{0}^{t_{1}} \tilde{p}_{j}^{M}(t) \tilde{\omega}_{j}(t) d t & =\int_{0}^{t_{1}}\left[\left(\tilde{p}_{j}^{D}(t)-\tilde{g}_{j}\left(\omega_{j}(t)\right)\right] \tilde{\omega}_{j}(t) d t\right. \\
\leq & \int_{0}^{t_{1}} \tilde{p}_{j}^{D}(t) \tilde{\omega}_{j}(t) d t \\
& \leq \sqrt{\int_{0}^{t_{1}}\left|\tilde{p}_{j}^{D}(t)\right|^{2} d t \int_{0}^{t_{1}}\left|\tilde{\omega}_{j}(t)\right|^{2} d t} \\
& \leq \int_{0}^{t_{1}} K_{j} \tilde{\omega}_{j}(t)^{2} d t<\int_{0}^{t_{1}} D_{j} \tilde{\omega}_{j}(t)^{2} d t
\end{aligned}
$$

where inequality (23a) follows from the fact that $g_{j}$ is a nondecreasing ${ }^{20}$ function of $\omega_{j}$, (23b) from the Cauchy-Schwarz inequality, and (23c) from inequality (22) and $K_{j}<D_{j}$.

Using (23), it is straightforward to show that

$$
\begin{aligned}
& \int_{0}^{t_{1}} D_{j} \tilde{\omega}_{j}(t)^{2} d t-\int_{0}^{t_{1}} \tilde{p}_{j}^{M}(t) \tilde{\omega}_{j}(t) d t \\
= & \int_{0}^{t_{1}} \tilde{s}_{j}^{G}(t)\left(-\tilde{\omega}_{j}(t)\right) d t \geq\left(D_{j}-K_{j}\right) \int_{0}^{t_{1}} \tilde{\omega}_{j}(t)^{2} d t \geq 0
\end{aligned}
$$

holds for all $j \in G$. Inequality (24) implies input strict passivity of the system with output $\tilde{s}_{j}^{G}=\tilde{p}_{j}^{M}-\tilde{d}_{j}^{u}$ and input $-\tilde{\omega}_{j}$, about the equilibrium point considered, since (24) implies from [31, Lemma 1] the existence of a positive definite storage function $V$ satisfying the local input strict passivity condition in Definition 2.

Before proving Corollary 1 we prove first a simpler result stated as Lemma 1 below.

\footnotetext{
${ }^{19} \mathrm{As}$ in the main text, for a variable $x$ that depends on time we use the notation $\tilde{x}$ to denote deviations from equilibrium, i.e. $\tilde{x}:=x-x^{*}$, where $x^{*}$ is the value of $x$ at the equilibrium point mentioned in the proposition.

${ }^{20}$ Note that $\tilde{g}_{j}\left(\omega_{j}\right) \tilde{\omega}_{j}=\left[g_{j}\left(\omega_{j}\right)-g_{j}\left(\omega_{j}^{*}\right)\right]\left[\omega_{j}-\omega_{j}^{*}\right] \geq 0$ since function $g_{j}\left(\omega_{j}\right)$ is nondecreasing with respect to $\omega_{j}$.
} 
Lemma 1: Consider the generation dynamics in (10) and let equation (11) hold. Then, for any equilibrium where (12) holds, the system with input $-\omega_{j}$ and output $s_{j}^{G}=p_{j}^{M}-d_{j}^{u}$ is globally input strictly passive about this equilibrium.

Proof of Lemma 1: The Lemma follows from Proposition 1 by showing that the $\mathcal{L}_{2}$-gain condition in the Proposition is satisfied with $g_{j}=0$. In particular, let $T_{j}(s)$ be the transfer function relating $\hat{\tilde{p}}_{j}^{c}(s)$ and $\hat{\tilde{p}}_{j}^{M}(s)$ in (10), given by

$$
T_{j}(s)=\frac{1}{\left(\tau_{g, j} s+1\right)\left(\tau_{b, j} s+1\right)}, j \in G .
$$

It is easy to show that $\sup _{\phi}|T(j \phi)|=1$, hence the system from $\tilde{p}_{j}^{c}$ to $\tilde{p}_{j}^{M}$ has $\mathcal{L}_{2}$-gain less than or equal to 1 (e.g. [22, p.18]). Using also equation (12) we thus have

$$
\left\|\tilde{p}_{j}^{M}\right\|_{2} \leq\left\|\tilde{p}_{j}^{c}\right\|_{2}<D_{j}\left\|\tilde{\omega}_{j}\right\|_{2} .
$$

With the choice $g_{j}=0$ we have $p_{j}^{M}=p_{j}^{D}$. It hence follows from (25) that the $\mathcal{L}_{2}$-gain condition in Proposition 1 holds, therefore Proposition 1 can be used to deduce input strict passivity of the system.

Proof of Corollary 1: The proof is analogous to that of Lemma 1, but we additionally show that by optimizing over a class of nonzero functions $g_{j}$ in Proposition 1, a less restrictive gain condition can be obtained.

In particular, we consider a variable $p_{j}^{D}$ of the form

$$
p_{j}^{D}=p_{j}^{M}-\hat{D}_{j} p_{j}^{c}
$$

where $\hat{D}_{j} \geq 0$ is a constant that will be appropriately chosen. Note that $p_{j}^{c}\left(\omega_{j}\right)$ is a nonlinear function of frequency that satisfies the condition on $g_{j}($.$) in Proposition 1, since it is$ equal to $k_{p_{i}^{M}}$ in (9a) and $\left(C_{j}^{\prime}\right)^{-1}$ is non decreasing due to the convexity of function $C_{j}$.

We now consider the system from $\tilde{p}_{j}^{c}$ to $\tilde{p}_{j}^{D}$, which has transfer function ${ }^{21}$

$$
T_{j}(s)=\frac{1}{\left(\tau_{g, j} s+1\right)\left(\tau_{b, j} s+1\right)}-\hat{D}_{j}
$$

Also let $\rho_{j}=\tau_{g, j} / \tau_{b, j}$ and note that $\left\|T_{j}\right\|_{\infty}:=\sup _{\phi}\left|T_{j}(j \phi)\right|$ depends only on $\rho_{j}$ and $\hat{D}_{j}$. We consider now the optimization problem

$$
\sup _{\rho \geq 0} \inf _{\hat{D}_{j} \geq 0} \sup _{\phi \in \mathbb{R}}\left|T_{j}(j \phi)\right|
$$

The solution to this problem is $1 / 1.5396$ with the optimizing variables given by

$$
\begin{gathered}
\phi=\sqrt{\frac{\sqrt{\rho_{j}^{3}\left(2 \hat{D}_{j}\left(\rho_{j}+1\right)-1\right)\left(2 \hat{D}_{j}\left(\rho_{j}+1\right)-\rho_{j}\right)}+\rho_{j}^{2}\left(2 \hat{D}_{j}-1\right)}{2 \hat{D}_{j} \rho_{j}^{3} \tau_{b, j}^{2}}}, \\
\hat{D}_{j}=\frac{\sqrt{\rho_{j}^{4}+14 \rho_{j}^{2}+1}+\rho_{j}^{2}-6 \rho_{j}+1}{4\left(\rho_{j}-1\right)^{2}} \\
\rho_{j} \rightarrow 1
\end{gathered}
$$

\footnotetext{
${ }^{21}$ Note that $p^{c}(\omega)$ is still a nonlinear function of frequency and no linearization is carried out in the proof.
}

where (27a) and (27b) give the optimal values of $\phi$ and $\hat{D}_{j}$ respectively for a given $\rho_{j}$, and (27c) gives the optimal value of $\rho_{j}$.

It hence follows from (26), that with $\hat{D}_{j}$ chosen as its optimal value in $(27 b),(27 c)$, the $\mathcal{L}_{2}$-gain of the system from from $\tilde{p}_{j}^{c}$ to $\tilde{p}_{j}^{D}$ is less than $1 / 1.5396$, for all values of $\rho_{j}$. Therefore if we choose the gain $K$ in (12) such that $K<1.5396 D$ we have that

$$
\left\|\tilde{p}_{j}^{D}\right\|_{2}<\left\|\tilde{p}_{j}^{c}\right\|_{2} / 1.5396<D_{j}\left\|\tilde{\omega}_{j}\right\|_{2} .
$$

Hence the $\mathcal{L}_{2}$-gain condition in Proposition 1 holds, and Proposition 1 can be used to deduce input strict passivity of the system.

Remark 13: It should be noted that the bound $K<$ $1.5396 D$ guarantees stability for any values of $\rho_{j}=\tau_{g, j} / \tau_{b, j}$. For specific values of $\rho_{j}$ this condition can be further relaxed by considering the solution to the optimization problem (26) for the corresponding value of $\rho_{j}$ considered.

\section{APPENDIX B}

In this appendix we provide the pre-disturbance conditions of the simulations in Section VII for the bus voltages, net injections and power transfers. It should be noted that due to losses, the net injections from Table II are different than the sum of the power flows from Table III. The pre-disturbance conditions are provided by the Power System Toolbox and are calculated by solving a corresponding load flow problem.

\begin{tabular}{|c|c|c|c||c|c|c|c|}
\hline $\begin{array}{c}\text { Bus } \\
\text { Number }\end{array}$ & $\begin{array}{c}\text { Voltage } \\
\text { (p.u.) }\end{array}$ & $\begin{array}{c}\text { Phase } \\
\text { (degrees) }\end{array}$ & $\begin{array}{c}\text { Injection } \\
\text { (p.u.) }\end{array}$ & $\begin{array}{c}\text { Bus } \\
\text { Number }\end{array}$ & $\begin{array}{c}\text { Voltage } \\
\text { (p.u.) }\end{array}$ & $\begin{array}{c}\text { Phase } \\
\text { (degrees) }\end{array}$ & $\begin{array}{c}\text { Injection } \\
\text { (p.u.) }\end{array}$ \\
\hline 1 & 1.059 & 6.615 & 2.527 & 35 & 1.014 & 2.533 & 0.000 \\
2 & 1.052 & 8.434 & 0.000 & 36 & 1.042 & -0.847 & 1.020 \\
3 & 1.033 & 5.432 & 3.220 & 37 & 1.029 & -6.805 & 60.000 \\
4 & 1.006 & 4.314 & 5.000 & 38 & 1.056 & 8.677 & 0.000 \\
5 & 1.007 & 5.254 & 0.000 & 39 & 1.006 & -8.442 & 2.670 \\
6 & 1.009 & 5.935 & 0.000 & 40 & 1.068 & 15.216 & 0.656 \\
7 & 1.000 & 3.664 & 2.340 & 41 & 0.999 & 44.489 & 10.000 \\
8 & 0.999 & 3.122 & 5.220 & 42 & 0.999 & 38.925 & 11.500 \\
9 & 1.039 & 2.579 & 1.040 & 43 & 1.015 & -7.606 & 0.000 \\
10 & 1.018 & 8.453 & 0.000 & 44 & 1.014 & -7.637 & 2.675 \\
11 & 1.014 & 7.596 & 0.000 & 45 & 1.018 & 2.525 & 2.080 \\
12 & 1.055 & 7.620 & 0.090 & 46 & 1.032 & 9.646 & 1.507 \\
13 & 1.016 & 7.785 & 0.000 & 47 & 1.074 & 7.363 & 2.031 \\
14 & 1.013 & 6.238 & 0.000 & 48 & 1.076 & 9.279 & 2.412 \\
15 & 1.017 & 6.142 & 3.200 & 49 & 1.012 & 12.881 & 1.640 \\
16 & 1.033 & 7.679 & 3.290 & 50 & 1.012 & 19.331 & 1.000 \\
17 & 1.036 & 6.585 & 0.000 & 51 & 1.022 & 6.523 & 3.370 \\
18 & 1.034 & 5.720 & 1.580 & 52 & 0.993 & 38.592 & 24.700 \\
19 & 1.050 & 12.274 & 0.000 & 53 & 1.045 & 10.853 & -2.500 \\
20 & 0.990 & 10.841 & 6.800 & 54 & 0.980 & 14.411 & -5.450 \\
21 & 1.033 & 10.314 & 2.740 & 55 & 0.983 & 16.440 & -6.500 \\
22 & 1.050 & 14.997 & 0.000 & 56 & 0.997 & 17.492 & -6.320 \\
23 & 1.045 & 14.708 & 2.480 & 57 & 1.011 & 16.014 & -5.052 \\
24 & 1.039 & 7.852 & 3.090 & 58 & 1.050 & 20.336 & -7.000 \\
25 & 1.060 & 9.698 & 2.240 & 59 & 1.063 & 22.564 & -5.600 \\
26 & 1.056 & 8.199 & 1.390 & 60 & 1.030 & 16.453 & -5.400 \\
27 & 1.043 & 6.314 & 2.810 & 61 & 1.025 & 20.788 & -8.000 \\
28 & 1.052 & 11.333 & 2.060 & 62 & 1.010 & 15.904 & -5.000 \\
29 & 1.051 & 13.970 & 2.840 & 63 & 1.000 & 18.347 & -10.000 \\
30 & 1.054 & 6.069 & 0.000 & 64 & 1.016 & 4.862 & -13.500 \\
31 & 1.057 & 8.630 & 0.000 & 65 & 1.011 & 0.000 & -35.914 \\
32 & 1.051 & 10.956 & 0.000 & 66 & 1.000 & 46.024 & -17.850 \\
33 & 1.056 & 7.473 & 1.120 & 67 & 1.000 & 39.785 & -10.000 \\
34 & 1.065 & 2.537 & 0.000 & 68 & 1.000 & 45.530 & -40.000 \\
\hline & & & & & & & \\
\end{tabular}

TABLE II. Pre-disturbance conditions for voltage, phase and net power injections for the IEEE 68-bus system.

\section{REFERENCES}

[1] A. Kasis, E. Devane, and I. Lestas, "On the stability and optimality of primary frequency regulation with load-side participation," $54^{\text {th }}$ IEEE Conf. Decision Control, Dec. 2015. 


\begin{tabular}{|c|c|c|c|c|c|}
\hline $\begin{array}{l}\text { Bus } \\
\text { from }\end{array}$ & $\begin{array}{c}\text { Bus } \\
\text { to }\end{array}$ & $\begin{array}{l}\text { Line Power Transfer } \\
\text { (p.u.) }\end{array}$ & $\begin{array}{l}\text { Bus } \\
\text { from }\end{array}$ & $\begin{array}{c}\text { Bus } \\
\text { to }\end{array}$ & $\begin{array}{c}\text { Line Power Transfer } \\
\text { (p.u.) }\end{array}$ \\
\hline 1 & 2 & -0.84 & 26 & 29 & -1.75 \\
\hline 1 & 30 & 1.51 & 28 & 29 & -3.33 \\
\hline 2 & 3 & 3.86 & 29 & 61 & -7.95 \\
\hline 2 & 25 & -2.20 & 9 & 30 & -3.68 \\
\hline 2 & 53 & -2.50 & 9 & 36 & 3.25 \\
\hline 3 & 4 & 1.03 & 9 & 36 & 3.25 \\
\hline 3 & 18 & -0.41 & 36 & 37 & 24.91 \\
\hline 4 & 5 & -1.30 & 34 & 36 & 6.08 \\
\hline 4 & 14 & -2.68 & 35 & 34 & 0.00 \\
\hline 5 & 6 & -4.68 & 33 & 34 & 6.11 \\
\hline 5 & 8 & 3.38 & 32 & 33 & 6.74 \\
\hline 6 & 7 & 4.40 & 30 & 31 & -2.66 \\
\hline 6 & 11 & -3.64 & 30 & 32 & -3.23 \\
\hline 6 & 54 & -5.45 & 1 & 31 & -2.38 \\
\hline 7 & 8 & 2.05 & 31 & 38 & -0.05 \\
\hline 8 & 9 & 0.20 & 33 & 38 & -0.52 \\
\hline 9 & 30 & -3.68 & 38 & 46 & -0.58 \\
\hline 10 & 11 & 3.66 & 46 & 49 & -2.09 \\
\hline 10 & 13 & 2.84 & 1 & 47 & -0.84 \\
\hline 10 & 55 & -6.50 & 47 & 48 & -1.44 \\
\hline 12 & 11 & -0.01 & 47 & 48 & -1.44 \\
\hline 12 & 13 & -0.08 & 48 & 40 & -5.30 \\
\hline 13 & 14 & 2.75 & 35 & 45 & 0.00 \\
\hline 14 & 15 & 0.06 & 37 & 43 & 0.54 \\
\hline 15 & 16 & -3.14 & 43 & 44 & 0.54 \\
\hline 16 & 17 & 2.26 & 44 & 45 & -2.49 \\
\hline 16 & 19 & -4.49 & 39 & 44 & -0.35 \\
\hline 16 & 21 & -3.61 & 39 & 45 & -2.32 \\
\hline 16 & 24 & -0.59 & 45 & 51 & -6.90 \\
\hline 17 & 18 & 1.99 & 50 & 52 & -11.39 \\
\hline 17 & 27 & 0.26 & 50 & 51 & 10.39 \\
\hline 19 & 20 & 1.78 & 49 & 52 & -3.74 \\
\hline 19 & 56 & -6.29 & 52 & 42 & -0.10 \\
\hline 20 & 57 & -5.03 & 42 & 41 & -1.60 \\
\hline 21 & 22 & -6.36 & 41 & 40 & 6.24 \\
\hline 22 & 23 & 0.61 & 31 & 62 & -5.00 \\
\hline 22 & 58 & -7.00 & 32 & 63 & -10.00 \\
\hline 23 & 24 & 3.71 & 36 & 64 & -13.50 \\
\hline 23 & 59 & -5.59 & 37 & 65 & -35.91 \\
\hline 25 & 26 & 0.91 & 41 & 66 & -17.85 \\
\hline 25 & 60 & -5.38 & 42 & 67 & -10.00 \\
\hline 26 & 27 & 2.53 & 52 & 68 & -40.00 \\
\hline 26 & 28 & -1.26 & 1 & 27 & 0.02 \\
\hline
\end{tabular}

TABLE III. Pre-disturbance power transfers for the IEEE 68-bus system.

[2] H. Lund, "Large-scale integration of optimal combinations of PV, wind and wave power into the electricity supply," Renewable Energy, vol. 31, no. 4 , pp. 503-515, 2006

[3] A. Ipakchi, and F. Albuyeh, "Grid of the future," IEEE Power and Energy Magazine, vol. 7, no. 2, pp. 52-62, March 2009.

[4] F. C. Schweppe, R. D. Tabors, J. L. Kirtley, H. R. Outhred, F. H. Pickel, and A. J. Cox, "Homeostatic utility control," IEEE Trans. Power App. Syst., vol. 99, no. 3, pp. 1151-1163, May 1980.

[5] J. A. Short, D. G. Infield, and L. L. Freris, "Stabilization of grid frequency through dynamic demand control," IEEE Trans. Power Syst., vol. 22, no. 3, pp. 1284-1293, Aug. 2007.

[6] D. J. Hammerstrom et al., "Pacific Northwest GridWise testbed demonstration projects, part II: grid friendly appliance project," Pacific Northwest Nat. Lab., Tech. Rep. PNNL-17079, Oct. 2007.

[7] B. J. Kirby, Spinning reserve from responsive loads. United States Department of Energy, 2003.
[8] D. Trudnowski, M. Donnelly, and E. Lightner, "Power-system frequency and stability control using decentralized intelligent loads," IEEE PES T\&D Conf. Exhib., pp. 1453-1459, May 2006.

[9] A. Molina-Garciá, F. Bouffard, and D. S. Kirschen, "Decentralized demand-side contribution to primary frequency control," IEEE Trans. Power Syst., vol. 26, no. 1, pp. 411-419, May 2010.

[10] A. R. Bergen and V. Vittal, Power Systems Analysis. Prentice Hall, 1999.

[11] J. Machowski, J. Bialek, and J. Bumby, Power System Dynamics: Stability and Control. John Wiley \& Sons, 2011.

[12] P. Kundur, Power System Stability and Control. McGraw-Hill New York, 1994.

[13] C. Zhao, U. Topcu, N. Li, and S. H. Low, "Design and stability of loadside primary frequency control in power systems," IEEE Trans. Autom. Control, vol. 59, no. 5, pp.1177-1189, 2014.

[14] C. Zhao, and S. H. Low, "Optimal decentralized primary frequency control in power networks," $53^{\text {rd }}$ IEEE Conf. Decision Control, pp. 2467-2473, Dec. 2014.

[15] S. Tripp and C. De Persis. Optimal frequency regulation in nonlinear structure preserving power networks including turbine dynamics: an incremental passivity approach, arXiv:1509.07617v1, Sep 2015.

[16] E. Mallada and S. H. Low,. Distributed Frequency-Preserving Optimal Load Control, IFAC World Congress, 2014.

[17] J. W. Simpson-Porco, F. Dörfler, and F. Bullo, "Synchronization and power sharing for droop-controlled inverters in islanded microgrids," Automatica, vol. 49, no. 9, pp. 2603-2611, Sep. 2013.

[18] S. Trip, M. Bürger, and C. De Persis. "An internal model approach to frequency regulation in inverter-based microgrids with time-varying voltages," 53 rd IEEE Conf. Decision Control, pp 223-228, Dec. 2014.

[19] E. Mallada, C. Zhao, and S. H. Low, "Optimal load-side control for frequency regulation in smart grids," $52^{\text {nd }}$ Allerton Conf., 2014.

[20] X. Zhang and A. Papachristodoulou, "A real-time control framework for smart power networks with star topology," Amer. Control Conf., 2013.

[21] E. Devane, A. Kasis, M. Antoniou, and I. Lestas, "Primary frequency regulation with load-side participation Part II: beyond passivity approaches," IEEE Transactions on Power Systems, 2016 (in press).

[22] J. C. Doyle, B. A. Francis, and A. Tannenbaum. Feedback control theory, vol. 1, Macmillan Publishing Company New York, 1992.

[23] F. Dörfler, M. Chertov, and F. Bullo, "Synchronization in complex oscillator networks and smart grids," PNAS, vol. 110, no. 6, pp. 20052010, 2013.

[24] H. K. Khalil. Nonlinear systems, Prentice Hall New Jersey, 1996.

[25] K. W. Cheung, J. Chow, and G. Rogers, Power System Toolbox, v. 3.0. Cherry Tree Scientific Software, 2009.

[26] A. Kasis, E. Devane, and I. Lestas, "Primary frequency regulation with load-side participation: stability and optimality (extended version of this manuscript)," arXiv:1602.02800, Feb. 2016. http://arxiv.org/abs/1602.02800

[27] G. Rogers, Power System Oscillations. Kluwer Academic, 2000.

[28] A. Molina-Garcia, F Bouffard, and D. S. Kirschen, "Decentralized demand-side contribution to primary frequency control," IEEE Trans. Power Syst., vol. 26, no. 1, pp. 411-419, 2011.

[29] Power Energy Society, 'https://ewh.ieee.org/soc/pes/dsacom/testfeeders', Accessed on:10/06/2016.

[30] S. Boyd and L. Vandenberghe, Convex optimization. Cambridge University Press, 2004.

[31] P. Moylan and D. Hill, "Stability criteria for large-scale systems," IEEE Trans. Autom. Control, vol. 23, no. 2, pp. 143-149, 1978. 